\title{
Title:
}

\section{Intrasexual cuticular hydrocarbon dimorphism in a wasp sheds light on hydrocarbon biosynthesis genes in Hymenoptera}

\section{Authors:}

Victoria C. Moris ${ }^{1,2,}$, Lars Podsiadlowski ${ }^{3,4}$, Sebastian Martin ${ }^{3,4}$, Jan Philip Oeyen ${ }^{3,5}$, Alexander Donath ${ }^{3}$, Malte Petersen ${ }^{3,6}$, Jeanne Wilbrandt ${ }^{3,7}$, Bernhard Misof ${ }^{3}$, Daniel Liedtke $^{8}$, Markus Thamm ${ }^{9}$, Ricarda Scheiner ${ }^{9}$, Thomas Schmitt ${ }^{10}$, Oliver Niehuis ${ }^{1, *}$

\section{Affiliations:}

1 Department of Evolutionary Biology and Ecology, Institute of Biology I (Zoology), Albert Ludwig University Freiburg, 79104 Freiburg, Germany

2 Laboratory of Molecular Biology \& Evolution (MBE), Department of Biology, Université Libre de Bruxelles, 1000 Brussels, Belgium

3 Centre for Molecular Biodiversity Research, Leibniz Institute for the Analysis of Biodiversity Change / ZFMK, Museum Koenig, Adenauerallee 160, 53113 Bonn, Germany

4 Institute of Evolutionary Biology and Ecology, University of Bonn, An der Immenburg 1, 53121 Bonn, Germany

5 Centre for Ecological and Evolutionary Synthesis, Department of Biosciences, University of Oslo

6 Max Planck Institute of Immunobiology and Epigenetics, Stübeweg 51, 79108 Freiburg, Germany

7 Leibniz Institute on Aging - Fritz Lipmann Institute, Beutenbergstraße 11, 07745 Jena, Germany

8 Institute of Human Genetics, University of Würzburg, Am Hubland, 97074 Würzburg, Germany

9 Department of Behavioral Physiology and Sociobiology, University of Würzburg, Am Hubland, 97074 Würzburg, Germany

10 Department of Animal Ecology and Tropical Biology Biocenter, University of Würzburg, Am Hubland, 97074 Würzburg, Germany 
ORCID IDs:

Victoria C. Moris: 0000-0002-7454-0400

Lars Podsiadlowski: 0000-0001-7786-8930

Sebastian Martin: 0000-0003-3171-4420

Jan Philip Oeyen: 0000-0002-0116-2234

Alexander Donath: 0000-0001-5618-0547

Malte Petersen: 0000-0001-7601-9873

Jeanne Wilbrandt: 0000-0002-0363-3837

Bernhard Misof: 0000-0003-4175-6798

Daniel Liedtke: 0000-0003-0934-7169

Markus Thamm: 0000-0003-0480-2206

Ricarda Scheiner: 0000-0002-7515-4253

Thomas Schmitt: 0000-0002-6719-8635

Oliver Niehuis: 0000-0003-4253-1849

Corresponding authors:

Victoria C. Moris: victoria.clara.moris@gmail.com

Oliver Niehuis: oliver.niehuis@biologie.uni-freiburg.de 


\section{Abstract:}

Cuticular hydrocarbons (CHCs) cover the cuticle of insects and serve as desiccation barrier and for chemical communication. While the main enzymatic steps of $\mathrm{CHC}$ biosynthesis are well understood, few of the underlying genes have been identified. Here we show how exploitation of intrasexual $\mathrm{CHC}$ dimorphism in a mason wasp, Odynerus spinipes, in combination with whole-genome sequencing and comparative transcriptomics facilitated identification of such genes. RNAi-mediated knockdown of twelve candidate gene orthologs in honey bees, Apis mellifera, confirmed nine genes impacting $\mathrm{CHC}$ profile composition. Most of them have predicted functions consistent with current knowledge of $\mathrm{CHC}$ metabolism. However, we found first-time evidence for a fatty acid amide hydrolase also influencing $\mathrm{CHC}$ profile composition. In situ hybridization experiments furthermore suggest trophocytes participating in $\mathrm{CHC}$ biosynthesis. Our results set the base for experimental $\mathrm{CHC}$ profile manipulation in Hymenoptera and imply that the evolutionary origin of $\mathrm{CHC}$ biosynthesis predates the arthropods' colonization of land. 


\section{Introduction}

Cuticular hydrocarbons ( $\mathrm{CHCs}$ ) are long-chained molecules that cover the cuticle of most, if not all, insects ${ }^{1}$. They function as important desiccation barrier and are thought to have fostered the transition from marine to terrestrial habitats in the lineage leading to Hexapoda ${ }^{2}$. CHCs additionally serve as semiochemicals for inter- and intraspecific communication ${ }^{2}$. In Hymenoptera, they play a particularly important role in establishing eusocialism by enabling kin, nest-mate and caste recognition ${ }^{2}$. They have also been exploited for chemical deception and chemical mimicry ${ }^{2}$. Identifying genes involved in $\mathrm{CHC}$ biosynthesis of Hymenoptera is of major interest, as it would open the door for CHC profile manipulation (e.g., via CRISPR-Cas9 or RNA interference) in behavioral, ecological, and socio-biological studies on Hymenoptera.

Most insects synthesize $\mathrm{CHCs}$ de novo from fatty acid precursors ${ }^{3}$ in oenocytes. These secretory cells of ectodermal origin are found in the fat body of all major insect lineages ${ }^{4}$. The main enzymatic steps of $\mathrm{CHC}$ biosynthesis are well understood and involve fatty acid synthases (FAS), fatty acid elongases (ELO), fatty acid desaturases (Desat), fatty acyl-CoA reductases (FAR), and cytochrome p450 decarbonylases

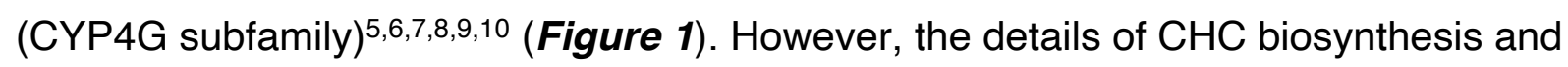
which genes encode the participating enzymes are not yet fully elucidated, particularly in the species-rich insect order Hymenoptera ${ }^{10}$. Furthermore, insights obtained from studying species of one insect lineage (e.g., Diptera) are not necessarily representative for species of a distantly-related lineage (e.g., Hymenoptera). Most genes associated with $\mathrm{CHC}$ biosynthesis belong to multi-gene families, and some of these exhibit remarkably high turnover rates (e.g., fatty acid desaturases ${ }^{11}$ ). Therefore, it is highly desirable to identify $\mathrm{CHC}$ biosynthesis-related genes in multiple insect lineages. A promising approach to this end is transcriptomic analysis comparing gene expression between samples of the same species that differ in their $\mathrm{CHC}$ composition from each other.

In most species, individuals of the same sex share the same set of CHCs, which is referred to as $\mathrm{CHC}$ profile type ${ }^{12}$. Intriguingly, previous research found females of the mason wasp Odynerus spinipes to be able to express two $\mathrm{CHC}$ profile types ${ }^{13}$. The 
two chemotypes (c1 and c2) qualitatively differ in $77 \mathrm{CHCs}$ from each other ${ }^{13}$. This $\mathrm{CHC}$ dimorphism offers an exceptional opportunity to identify genes involved in $\mathrm{CHC}$ biosynthesis, as $O$. spinipes females with different chemotypes do not appear to differ in any other trait from each other ${ }^{13,14}$. Hence, they should systematically differ only in the expression of $\mathrm{CHC}$ biosynthesis-related genes. $O$. spinipes females additionally show age-related quantitative changes in their $\mathrm{CHC}$ profile composition (Moris $\mathrm{V}$. et al., unpublished data), which can also be used to identify candidate genes.

In this study, we show how exploitation of the $\mathrm{CHC}$ dimorphism in 0 . spinipes females facilitated identification of $\mathrm{CHC}$ biosynthesis-related genes in Hymenoptera. Specifically, 1) we tested whether $O$. spinipes females keep a given chemotype during their adult life. 2) We sequenced, assembled, and annotated the genome of $O$. spinipes to aid accurate analysis of gene expression levels. 3) We identified candidate genes differentially expressed between $O$. spinipes females with different $\mathrm{CHC}$ composition. 4) We identified candidate gene orthologs in the honey bee (Apis mellifera) genome, a more trackable organism for in-depth molecular analyses. 5) We investigated which cell types of the worker honey bee fat body express the candidate genes. 6) We tested whether knockdown of selected candidate genes in worker honey bees via RNA interference (RNAi) impacts $\mathrm{CHC}$ profile composition.

\section{Results}

\section{Odynerus spinipes chemotype persistency}

None of the $\mathrm{O}$. spinipes females whose $\mathrm{CHC}$ profile we sampled multiple times during their adult life changed their chemotype (c1/c2): neither those kept under laboratory conditions $\left(\mathrm{N}_{\mathrm{c} 1}=10 ; \mathrm{N}_{\mathrm{c} 2}=17\right.$; sampled on average three times during the first 16 days after their eclosion; Supplementary Table 1), nor those studied in field populations $\left(N_{c 1}=8 ; N_{c 2}=10\right.$; sampled on average two times and with a time difference of on average five days; Supplementary Table 2). These results are consistent with the hypothesis that adult $O$. spinipes females cannot change their chemotype and justify 
associating $\mathrm{CHC}$ chemotype differences between females with gene expression differences between these females.

\section{Odynerus spinipes draft genome}

To foster comparative transcriptomic analyses on $O$. spinipes, we sequenced the genome of this species using short read sequencing technology. The assembly of the O. spinipes genome resulted in 21,922 scaffolds spanning a total of $171 \mathrm{Mb}, 89.5 \%$ of the total genome, which we estimated to be ca. $191 \mathrm{Mbp}$. The N50 value and L50 value of the draft genome assembly are 3,637 Mbp and twelve scaffolds, respectively. Exploiting whole body transcript libraries of one adult male and of two adult females (representing each chemotype) to foster annotation of nuclear-encoded protein-coding genes (Supplementary Table 3), we inferred a total of 16,677 gene models.

\section{Candidate genes in Odynerus spinipes}

We first identified statistically significant differentially expressed genes in transcriptomes of females with different chemotype in two batches of adult females that differed in sampling time (12-38 [batch 1] and 48-62 [batch 2] hours after eclosion) (Supplementary Tables 4-6). Considering only genes that were consistently differentially expressed in the two batches, we retained five candidate genes (Table 1; Supplementary Tables 7 and 8). We next searched for genes that were significantly differentially expressed in the two above batches of adult female wasps that differed in their age from each other, ignoring the wasps' chemotype. However, we only considered genes that belonged to four gene families known to be involved in $\mathrm{CHC}$ biosynthesis (i.e., FAS, ELO, Desat, FAR). This procedure resulted in the identification of eleven candidate genes (Table 1; Supplementary Table 9).

Combining the results from both approaches and accounting for redundancy (g7616 was identified with both search strategies), we identified a total of 15 candidate genes affecting $\mathrm{CHC}$ biosynthesis in $O$. spinipes (Table 1). Based on sequence homology, the candidate genes encode one fatty acid synthase, six fatty acid elongases, two fatty 
acid desaturases, three fatty acyl-CoA reductases, one fatty acid amine hydrolase, one parathyroid hormone-related peptide receptor-like protein, and one a rhythmically expressed gene 5 protein.

\section{Honey bee candidate gene orthologs}

We applied a phylogenetic approach to identify orthologs of twelve candidate genes belonging to multi-gene families (i.e., FAS, ELO, Desat, FAR) in the honey bee genome. The majority (ten) of the $O$. spinipes candidate genes share a 1:1 ortholog relationship to honeybee genes (Figures 2 and 3; Supplementary Figures 1 and 2; Table 1). However, the fatty acid desaturase candidate gene (g14712) of $O$. spinipes was found to have three co-orthologs in A. mellifera. We decided to consider all of them for further analyses (Table 1; Figure 3). We found one fatty acid desaturase candidate gene (g14708) to not have an ortholog in $A$. mellifera. We decided to include the phylogenetically most closely related $A$. mellifera gene for further experimental assessment (Table 1; Figure 3).

We applied the best reciprocal hit criterion to identify orthologs of the remaining three candidate genes (g283, g2290, g3059) in the honey bee genome (Table 1). In all instances except one we did find 1:1 orthologs. The exception was a gene predicted to encode a fatty acid amide hydrolase (FAAH) (g2290) that we found to be represented by three co-orthologs in the honey bee genome. Because this gene family had previously not been implicated with $\mathrm{CHC}$ biosynthesis, we considered only one ortholog (GB53695), that with the highest amino acid sequence similarity to g2290, in subsequent experiments.

We extended our list of candidate genes by seven genes (Table 2). Two of them are $A$. mellifera orthologs of genes shown to be involved in the $\mathrm{CHC}$ biosynthesis in the fruit fly Drosophila melanogaster (i.e., the honey bee ortholog of the two fatty acid synthases FASN1/FASN2, Supplementary Figure 1, and the honey bee ortholog of the three desaturases Desat 1, 2, and F, Figure 3). The remaining five genes encode fatty acid elongases that belong to clades phylogenetically closely related to genes 
known to be involved in $\mathrm{CHC}$ biosynthesis (Figure 2). The total number of candidate genes selected for further investigation in A. mellifera was thus 24 (Tables 1 and 2).

\section{Candidate gene expression sites}

In situ hybridization (ISH) experiments on fat body tissues from honey bee metasomas revealed 18 of the 24 candidate genes to be expressed in oenocytes: a fatty acid synthase (GB52590), eight fatty acid elongases (GB46038, GB54396, GB54397, GB51250, GB51247, GB54399, GB54404, GB55040), four fatty acid desaturases (GB40659, GB42218, GB48195, GB51236), three fatty acyl-CoA reductases (GB49380, GB50627, GB52087), one fatty acid amide hydrolase (GB53695), and one parathyroid hormone-related peptide receptor-like protein (GB52820) (Figure 4; Tables 1 and 2; negative controls performed with sense probes are shown in Supplementary Figure 3). Six of them (three fatty acid elongases, one fatty acid desaturase, one fatty acyl-CoA reductase, one fatty acid amide hydrolase) were found to be co-expressed in trophocytes, a fat body cell type different from oenocytes (Figure 4; Tables 1 and 2). We found two candidate genes, one fatty acid desaturase (GB51238) and one fatty acid elongase (GB54401), to be expressed in trophocytes only (Figure 4; Table 1). Three candidate genes, predicted to encode a fatty acid synthase (GB53412), a fatty acid elongase (GB40681), and a parathyroid hormonerelated peptide receptor-like protein (GB52820), respectively, were found to be expressed in cells located between the cuticle and the fat body layer (GB52820 was found also slightly expressed in oenocytes and trophocytes). We refer to these cells as hexagonal cells (Figure 4; Tables 1 and 2). In situ hybridization experiments on one candidate gene, predicted to encode a rhythmically expressed protein (GB44756), were inconclusive (marked by an asterisk in Figure 4), because the staining intensity of the treatment group did not differ from that of the negative controls (Supplementary Figure 3). We were unable to study cell-specific expression of one candidate gene (GB54302) encoding a fatty acid elongase, as its probe synthesis failed.

\section{Candidate gene knockdown experiments}


We assessed twelve candidate genes (selection criteria given in Supplementary Methods) for influencing $\mathrm{CHC}$ profile composition by conducting RNAi-mediated knockdown experiments on worker honey bees (Figures 5 and 6 ). Knockdown of nine candidate genes resulted in statistically significant changes of the $\mathrm{CHC}$ profile composition of the treated bees relative to control experiments in which we injected double-stranded RNA (dsRNA) of green florescent protein (GFP) (powered partial least squares discriminant analysis [PPLS-DA]: $p \leq 0.05$ after Benjamini-Hochberg correction for multiple testing). The nine genes were: four fatty acid elongases (GB51250, GB51274, GB54397, GB54404) (Figure 5), four fatty acid desaturases (GB40659, GB42218, GB48195, GB51238) (Figure 6), and one fatty acid amide hydrolase (GB53695) (Figure 5). CHC profile compositional changes associated with the knockdown of fatty acid desaturase GB51236 were statistically insignificant after Benjamini-Hochberg correction for multiple testing (PPLS-DA, $p=0.087$ ). RNAimediated knockdown of the remaining two genes, a fatty-acid synthase (GB52590) and a fatty-acyl-CoA reductase (GB52087), caused a premature death of the treated bees within the first two days after dsRNA injection.

While we found RNAi of the nine genes outlined above to generally result in a strong phenotypic effect 2-5 days after dsRNA injection, we did not detect statistically significant differences in expression levels of most target genes at the same point in time ( $p=0.126-0.607$; Welch's t-tests; Figures 5 and 6). The exceptions are three fatty acid desaturases (GB42218, GB48195, GB51238) that showed significantly lower expression levels in treated bees relative to control bees after Benjamini-Hochberg correction for multiple testing (GB42218: Wilcoxon signed-rank test, $W=122, p=$ 0.002; GB48195: Welch's t-test, $t(10.85)=4.01, p=0.010 ;$ GB51238: Welch's t-test, $t(9.67)=3.21, p=0.033$; Figure 6). Note that we were unable to measure the expression levels of GB54397 (encoding a fatty acid elongase) and of GB40659 (encoding a fatty acid desaturase), because the qRT-PCR oligonucleotide primers did not efficiently amplify a unique stretch of the genes' cDNA.

PPLS-DA of the CHC profiles provided information on the compounds that dominantly discriminate the RNAi treatment group and the control group. Knockdown 
of fatty acid elongases caused major $\mathrm{CHC}$ profile perturbations in several compound classes (Supplementary Figure 4), but it most notably impacted $\mathrm{CHC}$ chain lengths (Figure 5). Specifically, we found the chain lengths of $\mathrm{CHCs}$ positively correlating more than $50 \%$ along the first principal component of the PPLS-DA (Figure 5) with a fatty acid elongase dsRNA treatment (GB51247, GB51250, GB54397, GB54404) to be significantly lower than $\mathrm{CHC}$ chain lengths positively correlating with a control dsRNA treatment after correcting for multiple testing (Holm-Bonferroni) (GB51247: Welch's ttest, $t(12.04)=6.67, p<0.001 ;$ GB51250: Welch's t-test, $t(15.98)=6.18, p<0.001$; GB54397: Welch's t-test, $t(27.76)=3.54, p=0.003$; GB54404: Welch's t-test, $t(24.93)=$ 2.58, $p=0.016$ ) (Figure 7). We found the opposite pattern when interfering with the expression of GB53695 (encoding a fatty acid amide hydrolase): the CHC profiles of the treatment bees contained $\mathrm{CHCs}$ with significantly longer chain lengths than the $\mathrm{CHC}$ profiles of the control bees (Welch's t-test, $t(27.71)=-5.92, p<0.001$; Figures 5 and 7; Supplementary Figure 4).

Knockdown of fatty acid desaturases also caused major $\mathrm{CHC}$ profile perturbations and impacted the relative abundance of several compound classes (i.e., alkanes, alkenes, and methyl-branched alkanes) (Figure 6; Supplementary Figure 5). Depending on what gene we targeted with dsRNA, we found statistically significant differences in the relative abundance of alkenes with specific double bond positions between treated bees and control bees. Specifically, we found the relative abundance of alkenes with a double bond at even positions $(8,10)$ to be significantly smaller in bees treated with dsRNA of GB48195 (Wilcoxon signed-rank test, $W=87, p=0.022$ ) and of GB51238 (Welch's t-test, $t(11.94)=5.31, p=0.002$ ) than in control bees after correcting for multiple testing (Holm-Bonferroni) (Figure 8). We did not study the impact of target gene knockdown on alkadienes (with two double bonds), since the relative abundance of alkadienes was close to the detection limit of the used GC-MS instruments.

\section{Discussion}

\section{CHC biosynthesis-related genes in Hymenoptera}


Studying intrasexual $\mathrm{CHC}$ profile differences in the mason wasp 0 . spinipes provided promising candidate genes that modulate $\mathrm{CHC}$ profile composition in Hymenoptera. Knockdown experiments on twelve selected candidate genes in $A$. mellifera resulted in statistically significant $\mathrm{CHC}$ profile changes in nine of the experiments. In the light of these exciting results, the remaining candidate genes not further experimentally assessed in the present investigation are highly promising targets for future reverse genetic experiments.

\section{Fatty acid elongases}

Our study provided strong evidence for four fatty acid elongases (GB51247, GB51250, GB54397, GB54404) modulating CHC profile composition in Hymenoptera (Figures 5 and 7 ). The fact that we did not measure a statistically significant decrease in gene expression levels at the point in time when we sampled the bees' CHC profiles (Figure 5) could be due to a combination of two factors: 1) lag-time between gene expression level changes and $\mathrm{CHC}$ profile changes, and 2) short-term duration of the knockdown effect on the target genes' expression levels. Because the time points for sampling dsRNA-treated bees had been chosen based on when phenotypic changes became visible in a subset of samples (typically 2-5 days after dsRNA injection; Supplementary Table 10), they were possibly too late to still detect transient gene expression level changes. We consider the alternative explanation, namely that RNAi treatment did not have an effect on the target genes' expression levels, as unlikely, because of the observed phenotypic changes were in most instances consistent with the predicted function of the target genes.

Considering the gene tree, at least four major clades of fatty acid elongases seem to be involved in CHC biosynthesis in Hymenoptera: ELO B1, ELO B2, ELO B3, and ELO C7 (Figure 2). An additional clade (ELO C3; Figure 2) is known to be involved in $\mathrm{CHC}$ biosynthesis in $D$. melanogaster ${ }^{15,16}$. The wide distribution of known $\mathrm{CHC}$ biosynthesis-related genes and of candidate genes shown in this study to be expressed in oenocytes or trophocytes (see below) (Figure 2) within this gene family 
makes genes belonging to the clades ELO C1-7 promising targets for future knockdown experiments. In contrast, functional involvement of genes of clade ELO A in $\mathrm{CHC}$ biosynthesis currently appears unlikely, as the only gene of this clade functionally studied so far (i.e., baldspot of D. melanogaster) participates in spermatogenesis ${ }^{17}$.

Fatty acid elongases have been shown to catalyze the elongation of long fatty acyl$\mathrm{CoAs}^{2,10,18}$. Consistent with this idea, we found knockdown of fatty acid elongase genes to result in a decrease in the relative abundance of $\mathrm{CHCs}$ with chain lengths > 27 carbon atoms (Figures 5 and 7). Yet, we did not find any additional differences between the knockdown phenotypes. Lack of distinct phenotypes could be due to offtarget effects (i.e., knockdown of a gene coding for one fatty acid elongase simultaneously decreases the expression of another gene coding for a fatty acid elongase). However, except for two target genes, this scenario seems unlikely, as the nucleotide sequences of the synthesized dsRNAs (provided on zenodo: $10.5281 /$ zenodo.5552394) differed substantially from each other. The exception is GB51250 and GB54404, which differed only in 43\% of their nucleotides from each other. An alternative explanation for the lack of distinct phenotypic effects could be that the targeted fatty acid elongases exhibit, in stark contrast to those of mammals ${ }^{18}$, little substrate specificity. Finally, manipulation of the elongation steps could cause a shift of substrate concentrations along the entire $\mathrm{CHC}$ biosynthetic pathway, making it difficult to detect substrate specificity. Additional tools have to be used in future investigations to further interrogate the specific function and the substrate specificity of the enzymes encoded by the here identified genes (e.g., heterologous expression, as done by Pei et al. ${ }^{19}$, or in vivo synthesis using labeled precursors). The results of our study provide the foundation for such investigations.

\section{Fatty acid desaturases}

We identified four fatty acid desaturase genes (GB40659, GB42218, GB48195, GB51238) that modulate $\mathrm{CHC}$ composition in worker honey bees. Previous research on $D$. melanogaster had led to the identification of three closely related fatty acid 
desaturases (Desat1, 2, and F) for being involved in $\mathrm{CHC}$ biosynthesis and introducing double bonds in fatty acyl-CoA 20,21,22,23. All three desaturases belong to clade Desat A1 (Figure 3). In the honey bee, clade Desat A1 is represented only by a single gene (GB48195), which our knockdown experiments confirmed to be also involved in $\mathrm{CHC}$ biosynthesis in Hymenoptera. Intriguingly, the three additional fatty acid desaturases identified in our study to influence $\mathrm{CHC}$ profile composition in worker honey bee belong to two clades previously not associated with $\mathrm{CHC}$ biosynthesis (Figure 3): Desat A2 (GB42218, GB51238) and Desat B (GB40659). This demonstrates that fatty acid desaturases from very distantly related clades participate in $\mathrm{CHC}$ metabolism (i.e., Desat A1, Desat A2, Desat B; possibly also Desat D, which contains the O. spinipes candidate gene g14708, but which lacks an ortholog in $A$. mellifera).

Knockdown of the above-outlined four fatty acid desaturases had a strong effect in worker honey bees on both target gene expression (except GB40659) and on CHC profile composition (Figure 6). Moreover, we showed that knockdown of GB48195 and of GB51238 resulted in a decrease of alkenes, consistent with the idea of the encoded proteins introducing double bonds in fatty acyl-CoA. While all desaturases in $D$. melanogaster known to be involved in $\mathrm{CHC}$ biosynthesis (Desat1, 2, and F) show $\Delta 9$ specificity and thus facilitate synthesis of alkenes with double bonds at uneven positions ${ }^{20,22}$, GB48195 and GB51238 are the first fatty acid desaturases identified to be involved in the production of alkenes with double bonds at even positions ( 8 and 10) (Figure 8). Knockdown of the remaining two fatty acid desaturases (GB40659, GB42218) led to broad and complex compositional changes involving multiple CHCs. As discussed above, lack of distinct phenotypes could be due to off-target effects. However, this scenario seems also in this case unlikely, as the nucleotide sequences of the synthesized dsRNAs (provided on zenodo: 10.5281/zenodo.5552394) differed between all genes except two substantially from each other. The exception is GB42218 and GB51238, whose dsRNAs differed only in $29 \%$ of their nucleotides from each other. We consider off-target effects in this specific instance to unlikely have significantly impacted the results, however, as the nucleotide sequence used for antisense riboprobe (ISH) and the dsRNA probe (RNAi) caused different staining in the ISH experiments and different phenotypes in the knockdown experiments, 
respectively.

\section{Fatty-acid amide hydrolase}

We identified a fatty-acid amide hydrolase (GB53695) influencing CHC profile composition in worker honey bees. This result is remarkable, as fatty-acid amide hydrolases had not been associated with $\mathrm{CHC}$ biosynthesis before. Knockdown of GB53695, which we found to be expressed in oenocytes, resulted in a significant increase of the relative abundance of $\mathrm{CHCs}$ with chain lengths $>27$ carbon atoms (Figure 7). However, we did not detect statistically significant gene expression changes (Figure 5). Fatty-acid amide hydrolases are characterized by their amidase signature and are - in mammals - known to degrade fatty acid amides of endogenous lipids into fatty acids by removing the amine group ${ }^{24}$. How the functions of fatty-acid amide hydrolases relate to the modulation of $\mathrm{CHC}$ profiles in Hymenoptera remains unclear at this point. We deem it possible that the studied fatty-acid amide hydrolase impacts the availability of $\mathrm{CHC}$ precursors, by, for instance, releasing shortchain fatty acids that are then possibly reduced to aldehydes, which could be transformed to $\mathrm{CHCs}$ by a cytochrome p450 decarbonylase (Figure 1).

\section{Fatty acid synthases and fatty acyl-CoA reductases}

Knockdown of candidate genes encoding a fatty acid synthase (GB52590) and a fatty acyl-CoA reductase (GB52087) resulted in a premature death of the treated bees. A similar observation was made by other researchers when targeting genes of these two families in various insects (e.g., 25,26,27,28,29). We hypothesize that interfering with the expression of genes of the two gene families reduced - due to their central role in $\mathrm{CHC}$ biosynthesis - the overall availability of $\mathrm{CHCs}$ to an extend that it caused desiccation-related death of the treated bees ${ }^{26,28,29}$. Knockdown of fatty acid synthase could additionally have reduced the synthesis of other molecules fundamentally important for survival (e.g., cuticular lipids ${ }^{26}$ ). Despite the fact that our RNAi experiments on GB52590 and GB52087 remained inconclusive, alternative approaches should consider them highly promising candidate genes, as multiple lines 
of evidence strongly suggest both genes to be involved in $\mathrm{CHC}$ biosynthesis: a) ortholog relationship to $D$. melanogaster genes known to be involved in $\mathrm{CHC}$ biosynthesis (i.e., GB52590, ortholog of FASN328 fatty acid synthase which belongs to clade FAS B2; Supplementary Figure 1; GB52087, ortholog of the three fatty acylCoA reductases CG10097, CG13091, and CG1756016, all of which belong to clade FAR F; Supplementary Figure 2), b) expression in honey bee oenocytes (Figure 4), C) statistically significant expression differences of their $O$. spinipes orthologs (i.e, g3158, ortholog of GB52590; g1571, ortholog of GB52087) between 12-38 hours-old O. spinipes females with different chemotype (Supplementary Tables 5 and 7 ) and between females differing in age from each other (Table 1; Supplementary Table 9). Two other FAR-encoding candidate genes, GB50627 (clade FAR F) and GB49380 (clade FAR C) also seem promising candidate genes, as they are both expressed in honey bee oenocytes (Figure 4) and their O. spinipes orthologs (g2413 and g7842) were found to be differentially expressed between females differing in age from each other (Table 1; Supplementary Table 9).

\section{Evolutionary roots of CHC biosynthesis}

The phylogenetic distribution of genes involved in $\mathrm{CHC}$ biosynthesis across Euarthropoda (i.e., Chelicerata, Myriapoda, "Crustacea", and Hexapoda) has implications for our understanding of the evolution of $\mathrm{CHC}$ biosynthesis. All major gene families (Figure 1) and most subclades within them harboring genes known to be involved in CHC biosynthesis (FAS A, ELO C, ELO B, Desat A1, and potentially FAR C) are present in the extant major lineages of Euarthropoda. CHCs and oenocytes have also been reported from all major Euarthropoda lineages $30,31,32,33,34,35,36,37$. We therefore consider it reasonable to hypothesize that the capacity of $\mathrm{CHC}$ biosynthesis evolved before the divergence of the major lineages of Euarthropoda and, consequently, before the colonization of land. The only gene whose phylogenetic distribution is currently not fully consistent with this hypothesis is the cytochrome p450 decarbonylase (CYP4G gene family) (Figure 1) that catalyzes the final step of $\mathrm{CHC}$ biosynthesis $^{8,38}$ and which has - so far - only been found in Hexapoda ${ }^{38,39}$. As the 
cytochrome p450 gene family is known for its high turnover rate ${ }^{38}$, we do not put too much weight in this single exception.

\section{Possible importance of trophocytes for CHC biosynthesis}

We found almost all genes whose knockdown resulted in a $\mathrm{CHC}$ profile change to be expressed in oenocytes - consistent with the common view that $\mathrm{CHC}$ metabolism occurs in oenocytes (e.g., ${ }^{8,40,41,42}$ ). A major exception was GB51238, encoding a fatty acid desaturase. We found this gene exclusively and highly expressed in trophocytes (Figure 4), another cell type of the insects' fat body. While we cannot exclude the possibility that the gene is expressed at a very low level in oenocytes, we deem the result noteworthy, as other researches previously already suggested fat body cells other than oenocytes to be possibly involved in $\mathrm{CHC}$ biosynthesis ${ }^{28,43}$. Given the possible implications of $\mathrm{CHC}$ biosynthesis involving additional types of fat body cells, we recommend future studies to not exclusively focus on oenocytes when studying $\mathrm{CHC}$ metabolism.

\section{Molecular basis of the $\mathrm{CHC}$ profile dimorphism in 0 . spinipes females}

The knockdown experiments on $A$. mellifera helped better understanding the molecular basis for chemotype differences in $O$. spinipes females. $O$. spinipes females with chemotype 2 differ from those with chemotype 1 primarily by having alkenes with double bonds at even chain positions (8, 10, 12, and 14; chemotype 1 females have them at uneven chain positions) and by featuring larger quantities of alkenes with chain lengths $>25$ carbon atoms (Supplementary Figure 6). Intriguingly, knockdown of one (GB51238) of the three co-orthologs of fatty acid desaturase g14712 caused a decrease in the relative abundance of alkenes with double bonds at even chain positions in worker honey bees. The phenotypic change is thus fully consistent with the idea that higher expression of the gene's ortholog (g14712) in O. spinipes females with chemotype 2 gives rise to a higher abundance of alkenes with double bonds at even chain positions. Likewise, knockdown of the fatty acid elongase (g7616) ortholog 
in worker honey bees (GB51247) caused a decrease of the relative abundance of $\mathrm{CHCs}$ with chain lengths larger than 27 carbon atoms. The result is in line with the idea that higher expression of the gene's ortholog (g7616) in 0 . spinipes females with chemotype 2 gives rise to a higher abundance of alkenes with chain lengths $>25$ carbon atoms. It is remarkable that, considering the large number of qualitative and quantitative differences between the two $O$. spinipies $\mathrm{CHC}$ chemotypes ${ }^{13}$, most of the differences seem to be due to the action of only two enzymes in the $\mathrm{CHC}$ biosynthesis pathway.

While comparative analysis of gene expression levels between $O$. spinipes females with different chemotypes led to the identification of additional candidate genes than the above-discussed fatty acid elongase and fatty acid desaturase (Table 1), their role in shaping the two distinct phenotypes remains unclear. For example, we found a fatty acid amid hydrolase (g2290) being more expressed in females with chemotype 2 than in females with chemotype 1. However, knockdown of one of the three co-orthologs (GB53695) in the worker honey bees caused a decrease of short chain CHCs, contrary to what we would have predicted from the $\mathrm{CHC}$ profile characteristics of $O$. spinipes females with chemotype 2. The predicted functions of other candidate genes (e.g., rhythmically expressed gene 5 protein [g283]; parathyroid hormone or parathyroid hormone-related peptide receptor-like protein [g3059]) also provide no clue on how they could contribute to the observed $\mathrm{CHC}$ chemotype differences.

Our study demonstrates how comparative transcriptomic analyses on a non-model organism featuring unique characteristics can deliver valuable information that research on established model organisms may not be able to provide. It furthermore enables future experimental manipulation of $\mathrm{CHC}$ profiles in Hymenoptera and subsequent functional investigations on the activity and substrate specificity of the identified genes. 


\section{Methods and Material}

\section{Odynerus spinipes chemotype persistency analysis}

\section{Lab experiments}

We collected prepupae of $O$. spinipes females and males from trap nests (details in Supplementary Methods). Each female was placed in a separate observation cage (30 cm x $30 \mathrm{~cm} \times 30 \mathrm{~cm}$, Bioform, Nürnberg, Germany) and provided with a conspecific male and absorbent paper soaked with honey and water. The presence of males was meant to simulate field conditions for the females. The cages were kept in a climate chamber $\left(70 \%\right.$ humidity, $23{ }^{\circ} \mathrm{C}$ during day, $18{ }^{\circ} \mathrm{C}$ during night, and with a $12 / 12 \mathrm{~h}$ day/night cycle). We sampled the cuticular hydrocarbons (CHCs) of 23 females with the aid of Solid Phase Micro-Extraction (SPME) fibers (see below) at two days, seven/eight days, and 14 days (or immediately after they died) after they had eclosed (Supplementary Table 1).

\section{Field experiments}

We sampled the CHCs of 18 females two to four times during their adult life in the field (Supplementary Table 2). Females were caught at their nest entrance, color-coded with water resistant paint markers (Edding 780, $0.8 \mathrm{~mm}$, Ahrensburg, Germany), CHCsampled with the aid of SPME fibers, and released the next morning at their nest site. The sampling time coincided with the start of nest building activity.

\section{Cuticular hydrocarbon extraction}

CHCs from living wasps were sampled by stroking a SPME fiber (Supelco, coating: polydimethylsiloxane, $100 \mu \mathrm{m}$, Sigma-Aldrich, Bellefonte, PA, USA) for $2 \mathrm{~min}$. (field populations) or 4 min. (lab population) over the cuticle of a female's metasoma. Wasps processed in 2016 were anesthetized by exposing them for $1 \mathrm{~min}$. to $\mathrm{CO}_{2}$, those 
processed in 2017 by cooling them down for $3 \mathrm{~min}$. at $-20{ }^{\circ} \mathrm{C}$ (because we suspected $\mathrm{CO}_{2}$ treatment to cause amnesia in the treated wasp, as wasps treated this way in 2016 rarely returned to their nest; see ${ }^{44}$ ).

\section{Gas chromatography and mass spectrometry}

All $\mathrm{CHC}$ extracts were either analyzed with a gas chromatograph coupled to a mass spectrometer (GC-MS) or with a gas chromatograph-flame ionization detector equipped with a SLB-5 MS non-polar capillary (GC-FID) applying the same temperature profile (details in Supplementary Methods). The GC-MS data were recorded and quantified with the software HP Enhanced ChemStation G1701AA (version A.03.00; Hewlett Packard, Palo Alto, California, USA). The GC-FID data were recorded and quantified with the software GCSolution (Shimazu, Wemmel, Belgium). The chemotype of the female wasps was inferred from the presence/absence of chemotype-specific alkenes ${ }^{13,14}$ with diagnostic Kovats indices ${ }^{45}$.

\section{Odynerus spinipes draft genome}

\section{NGS library preparation and sequencing}

We sequenced the nuclear genome at a base-coverage depth of ca. 86x using Illumina HiSeq2000 sequencing technology and three different types of NGS libraries: $250 \mathrm{bp}$ paired-end [10.58 Gbp] (55x), 800 bp paired-end [2.45 Gbp] (13x), and 3 kbp matepair [3.4 Gbp] (18x), generated from a single pool of DNA extracted from males and

females (details in Supplementary Methods). To foster gene annotation, we sequenced whole-body transcriptomes of one adult male and of two adult females (one of each chemotype). DNA and RNA extraction, library preparation, and Illumina DNA sequencing were done by BGI Tech Solutions (Hongkong).

Genome assembly and annotation 
All raw reads of genomic DNA were processed with trimmomatic ${ }^{46}$ version 0.33 (details in Supplementary Methods). The processed reads were then used to assemble the genome with Platanus ${ }^{47}$ version 1.2.4 using all three libraries in each of the three assembly steps (contig construction, scaffolding, and gap closing) and applying the software's default parameters. The protein-coding gene repertoire was annotated with the BRAKER $a b$ initio gene prediction pipeline ${ }^{48}$ version 2.1 and providing the RNAseq data of the three above-mentioned adult wasps as intrinsic evidence (details in

\section{Supplementary Methods).}

\section{Comparative gene expression analysis}

\section{Odynerus spinipes samples}

We sequenced metasoma transcriptomes of twelve $O$. spinipes females raised under laboratory conditions from field-collected prepupae (Supplementary Table 4): six of them (three of each chemotype) were sampled 12-38 $\mathrm{h}$ after they had eclosed, the other six (three of each chemotype) were sampled 48-62 h after they had eclosed. The prepupae were collected from three field sites (Supplementary Table 4).

Prior to RNA fixation, the eclosed females were kept separate from each other at 21 ${ }^{\circ} \mathrm{C}$ in polystyrene tubes (53 mm x $100 \mathrm{~mm}$, Bioform, Nuremberg, Germany) containing moistened cotton and experienced a 12/12 h day/night cycle. All samples were euthanized at the same time of the day $(1 \mathrm{pm})$ to minimize a potential impact of expression differences by rhythmically expressed genes (note that expression of Desat1 follows such a diurnal rhythm in $D$. melanogaster ${ }^{49}$ ). Wasps were euthanized by submersing them for 60-120 sec. in hexane ( $n$-hexane for gas chromatography, SupraSolv, Merck, Germany). The CHC extracts were used to infer the wasps' chemotypes as described above. Each wasp's metasoma was ground with a sterile pestle in $1 \mathrm{~mL}$ RNAlater (QIAGEN, Hilden, Germany) and kept at $-80^{\circ} \mathrm{C}$ before further processing it.

Transcriptome sequencing 
RNA extraction, NGS library preparation, and 150 bp paired-end nucleotide sequencing with an Illumina NextSeq 500 (Illumina, San Diego, USA) were done by StarSEQ (Mainz, Germany) using standard protocols. We collected on average 20-44 million reads per sample.

\section{Gene expression analysis}

The RNA-seq data were analyzed with software integrated in the Galaxy Europe platform (https://usegalaxy.eu). We quality-trimmed the raw reads and removed NGS library adapters with Trim Galore version 0.4.3.0 (http://www.bioinformatics.babraham.ac.uk/projects/trim galore/). The quality of the $\begin{array}{lllllll}\text { raw reads was assessed with } & \text { FastQC version } 0.67\end{array}$ (http://www.bioinformatics.babraham.ac.uk/projects/fastqc/). The gene model file (GTF) of the $O$. spinipes genome was used to model splice junctions with the software $\mathrm{STAR}^{50}$ version 2.5.2b0 via mapping the transcript raw reads onto the genome assembly. We inferred a matrix of normalized read counts per gene, considering the genes in the above GTF file, with featureCounts ${ }^{51}$ version 1.5.3.

Because our initial experimental design included only one batch of six transcriptomes (three of each chemotype) (Supplementary Table 4), we applied a two-step procedure for identifying genes differentially expressed between females with different chemotypes: we first searched with DESeq2 ${ }^{52}$ version 2.11.39 for differentially expressed genes in each of the two batches, using a False discovery rate (FDR) of 0.05 as cutoff. We then reciprocally assessed genes that this analysis indicated to be differentially expressed between the two chemotypes in the other batch, this time applying a more conservative Holm-Bonferroni correction for multiple testing. Only genes that were significantly differentially expressed in both analyses were kept as candidate genes. We assessed with edge ${ }^{53}$ version 3.28 .0 in the $R$ statistical software version 3.4.1 (http://www.R-project.org) whether the five genes identified with the above-described procedure were also found differentially expressed in the two batches ( $p$-value $<0.05$ after Holm-Bonferroni correction for five tests). We used 
DESeq2 as well as edgeR to assess statistical significance of gene expression differences between $O$. spinipes females of different age classes, ignoring the wasps' chemotype. We considered genes, judged to differ in their expression by either of the two programs, as candidates if they encoded fatty acid synthases (FAS), fatty acid elongases (ELO), fatty acid desaturases (DESAT), or fatty acyl-CoA reductases (FAR).

\section{Identification of candidate gene orthologs in the honey bee genome}

Honey bee orthologs of $O$. spinipes candidate genes belonging to the gene families Desat, ELO, FAR, and FAS were identified by their phylogenetic position in the corresponding gene trees. To this end, we identified the proteins of the four gene families by searching for proteins with the protein family-characteristic protein domains (see below) available as HMM models from PFAM database ${ }^{54}$ (PFAM-A, version 28). The HMM models were searched with the aid of hmmer ${ }^{55}$ version $3.1 \mathrm{~b} 1$ against the protein sets of 37 Euarthropoda, including 0 . spinipes, with sequenced genomes (details in Supplementary Methods). To identify fatty acid synthases, we searched for proteins containing the PFAM domains ketoacyl-synt KS (Pfam family accession PF00109), acyl-transferase-1 (PF00698), and PS-DH (PF14765) (e-value < 1e-5). To identify fatty acid elongases, we searched for proteins containing the ELO PFAM domain PF01151 (e-value < 1e-30), and consisting at least of 200 amino acids. To identify fatty acid desaturases, we searched for proteins containing the PFAM domain PF00487 (e-value < 1e-20). Note that we did not consider sphingolipid desaturases, as they show little amino acid sequence similarity to other desaturases (see ${ }^{11}$ ). To identify fatty acyl-CoA reductases, we searched for the simultaneous presence of the two PFAM domains PF07993 (e-value < 1e-30) and PF03015 (e-value < 1e-20). The amino acid sequences of a given gene family were aligned with MAFFT ${ }^{56}$ version 7.123 and phylogenetically analyzed with the software IQ-TREE57 version 1.6 (details in

\section{Supplementary Methods).}

Honey bee orthologs of the remaining $O$. spinipes candidate genes and which did not belong to the above gene families were identified by applying the best reciprocal hit criterion and searching the protein repertoires of the two species with the blastp 
program of the BLAST+ software suite ${ }^{58,59}$.

\section{In situ hybridization}

Synthesis of sense and anti-sense riboprobes

RNA probes were synthesized according to standard protocols ${ }^{60}$ from cloned partial mRNA sequences of all candidate genes (Tables 1 and 2) using DIG and FLU RNA Labeling Kits (Roche, Basel, Switzerland). Strand cDNA was synthesized from isolated total RNA (1 $\mu \mathrm{g}$, DNase-treated) of fat body tissues isolated from $A$. mellifera using the Quantitect kit (QIAGEN GmbH, Hilden, Germany). The resulting cDNA library was used to amplify target regions via polymerase chain reactions (PCRs) (Mastercycle DNA Engine Thermal cycler PCR, Eppendorf AG, Hamburg, Germany) using the Phusion high fidelity kit (New England Biolabs, Ipswich, MA, USA). Amplicons were cloned with the NEB PCR Cloning kit (New England Biolabs GmbH, Ipswich, MA, USA), and the plasmids were extracted using the Gen Elute Plasmid Miniprep kit (Sigma-Aldrich, Steinheim, Germany) and were sent to Genewiz (Genewiz, Leipzig, Germany) for nucleotide sequencing. The cloned gene fragments (387-774 bp long) were used to synthesize anti-sense and sense RNA probes using T7 (Roche, Mannheim, Germany) or SP6 (Roche, Mannheim, Germany) RNA polymerases. Sense probes served as negative control to each anti-sense probe. Oligonucleotide primers used for probe cloning were designed with the online software Primer-BLAST61 and synthesized by Metabion (Planegg, Germany) or Sigma-Aldrich (Steinheim am Albuch, Germany) (Supplementary Table 11).

\section{Whole mount RNA in situ hybridization}

In situ hybridization was done on worker honey bees collected from a single hive at Würzburg University. All bees eclosed 4-8 days prior to their processing, were reared from prepupae in climate chambers at $28^{\circ} \mathrm{C}$, and were collectively kept in observation cages at $32{ }^{\circ} \mathrm{C}$ with $30 \%$ sugar solution. Metasoma tergites and sternites were 
dissected from living bees (placed on ice for $5 \mathrm{~min}$ ) in $4 \%$ paraformaldehyde (PFA) (Sigma-Aldrich, Steinheim am Albuch, Germany; dilution in Phosphate buffered saline [PBS]). The dissected segments were fixed overnight in a solution consisting of $2 \mathrm{~mL}$ heptane (Carl Roth, Karlsruhe, Germany) and $2 \mathrm{~mL}$ of $4 \%$ PFA on a rotator at room temperature. The fixation was finalized following the protocol given by Dearden et al. ${ }^{62}$. Whole-mount RNA in situ hybridization was done as described by Dearden et al. ${ }^{63}$, with minor modifications outlined in Supplementary Methods. All mounting media included DAPI (0.5 $\mu \mathrm{g} / \mathrm{mL}$; Thermofisher Scientific, Waltham, Massachusetts, USA) to achieve nuclear counterstaining. High resolution images of stained tissues mounted on glass slides were obtained with a Zeiss Axio Imager A1 fluorescence microscope, a Zeiss AxioCam MRc, DAPI fluorescence filters, a Zeiss HXP120 UV light unit, and the Zeiss Axiovision software (Carl Zeiss Jena $\mathrm{GmbH}$, Jena, Germany). Overlay of brightfield images and DAPI fluorescence was done with ImageJ/Fiji (https://fiji.sc/). Trophocytes were distinguished from oenocytes by their polyploid or polygonal cells (oenocytes are round) with a large irregular nucleus (the nucleus of oenocytes is round or oval) $)^{64}$.

\section{RNAi-mediated knockdown experiments}

dsRNA synthesis

Double-stranded RNA (dsRNA) was synthesized in vitro from 387-774-bp-long inserts - previously generated for RNA probe synthesis - using MEGAscript RNAi kit (Ambion, Texas, USA) (details in Supplementary Methods). T7 promotor was added to the oligonucleotide primers (listed in Supplementary Table 11) used to amplify the plasmids. A plasmid with a DNA fragment encoding green florescent protein (GFP), kindly provided by Anne-Kathrin Rohlfing (nucleotide sequence on zenodo: 10.5281/zenodo.5552394), was used to synthesize dsRNA that served as negative control. We verified that the GFP nucleotide sequence had no significant match in the honey bee genome ${ }^{65}$ by searching it with tools of the BLAST+ software suite 58,59 against the honey bee genome (GCF_000002195.4_Amel_4.5). The identity of the 
amplicons was verified by nucleotide-sequencing them. All dsRNA probes were diluted in MOPS Ringer solution (270 mM NaCl, $3.2 \mathrm{mM} \mathrm{KCl,} 1.2 \mathrm{mM} \mathrm{CaCl}_{2}, 10 \mathrm{mM} \mathrm{MgCl}$, $10 \mathrm{mM}$ Y-(N-Morpholino) propane sulfonic acid (MOPS), ph 7.4) to reach a final concentration of $5 \mu \mathrm{g} / \mu \mathrm{L}$.

\section{$R N A i$}

Knockdown experiments were done on worker honey bees reared as described above and chilled prior to the dsRNA injection. All bees of a given experiment came from the same hive, eclosed 6-7 days prior to dsRNA treatment and belonged to the same caste (workers, nurse bee). We injected between the $2^{\text {nd }}$ and $3^{\text {rd }}$ tergite of each bee's the metasoma $1 \mu \mathrm{L}$ dsRNA (5 $\mu \mathrm{g}$ ) with a Hamilton microliter syringe (MICROLITER Series 7000 Tip type 2, $7105 \mathrm{KH}$, Carl Roth, Karlsruhe, Germany). Per experiment, we treated 6-12 bees with dsRNA of the target gene of interest and 6-13 bees with dsRNA of GFP, which served as control (Supplementary Table S11). Bees of different experiments were kept in separated observation cages and were fed with fed $30 \%$ sugar water. Note that we were forced to use the same control group when assessing knockdown of GB51250 and GB48195 (which have been carried out at the same time and with bees from the same hive), as we lacked bees to make an extra control group at that specific time.

Analysis of $\mathrm{CHC}$ profile changes

After dsRNA injection, we daily sampled the CHCs of six bees (three of the treatment group and three of the control group) from a given RNAi experiment with SPME fibers, probing each bee's metasoma. As soon as notable $\mathrm{CHC}$ profile differences between treatment bees and control bees were detected (sampling times given in Supplementary Table 10), all samples from the corresponding RNAi experiment were euthanized by immersing them for $2 \mathrm{~min}$. in hexane ( $\mathrm{n}$-hexane for gas chromatography, SupraSolv, Merck, Germany) and were subsequently frozen in liquid nitrogen. The bees' metasoma were subsequently stored at $-80^{\circ} \mathrm{C}$ before further processing them. 
CHC extracts were analyzed with a GC-MS (details in Supplementary Methods). $\mathrm{CHC}$ abundance values were normalized by dividing them by the total abundance of CHCs identified in a given $\mathrm{CHC}$ extract (available on zenodo: 10.5281/zenodo.5552394). The normalized data were analyzed in Rstudio ( $R$ version 4.0.3), using functionalities from the following packages: RVAideMemoire version 0.980 (https://CRAN.R-project.org/package=RVAideMemoire), vegan version 2.5-7, Hotelling version 1.0-7, pls version 2.7-3, FactoMineR ${ }^{66}$ version 2.4, and Factoextra version 1.0.6 (https://CRAN.R-project.org/package=factoextra). The statistical significance of $\mathrm{CHC}$ profile differences between groups was assessed with a powered partial least squares discriminant analysis and applying pairwise permutation tests ${ }^{67,68}$. Differences in chain lengths and the relative abundance of specific alkenes were assessed with Welch's t-test or with the Wilcoxon signed-rank test, depending on whether or not the data appeared normally distributed (assessed with the Shapiro-Wilk test), using the stats package version 3.6.2 in Rstudio. $P$-values were corrected with the Holm-Bonferroni method for multiple testing. Boxplots were drawn with the ggplot2 package (https://ggplot2.tidyverse.org) in Rstudio.

\section{Analysis of gene expression changes}

We extracted the total RNA from the metasoma of each bee treated with dsRNA using RNeasy Plus Mini kit (QIAGEN, Hilden, Germany) and quantified the relative expression of the target genes via real-time quantitative PCR (RT-qPCR) using the QuantiTect SYBR Green RT-PCR kit (QIAGEN, Hilden, Germany) and a StepOnePlus Real-Time PCR System (Applied Biosystems-Life technologies, ThermoFisher Scientific, Waltham, Massachusetts, USA). We quantified expression levels in at least five samples per group (i.e., treatment and control) and in three technical replicates per sample. RT-qPCR oligonucleotide primers used to assess the target genes' expression levels are listed in Supplementary Table 12 and were designed with Primer-BLAST ${ }^{61}$. If possible, oligonucleotides were designed so that the resulting amplicon was 150-250 bp in length, that the oligonucleotides' melting temperature was between 57 and $63^{\circ} \mathrm{C}$, that the oligonucleotides would span exon-exon junctions, that 
the amplified region was localized at the 5' to the dsRNA target region ${ }^{69}$, and that the amplified region was not overlapping with the dsRNA target region. We used GB49975 (predicted ribosomal protein L1) as reference gene (Supplementary Table 11), because its $O$. spinipes ortholog was one of the genes that exhibited the least expression differences between females with different chemotype. All oligonucleotide primer pairs exhibited an amplification efficiency of more than 95\%, and each amplicon showed a single melting temperature maximum. The expression data were analyzed following the protocol given by Pfaffl, $2001^{70}$. Gene expression levels were statistically assessed with the Welch's t-test and with the Wilcoxon signed-rank test, as described above. $P$-values were corrected with the Benjamini-Hochberg method for multiple testing. 


\section{References}

1. Lockey, K. H. Lipids of the insect cuticle: origin, composition and function. Comp. Biochem. Physiol. B: Comp. Biochem. 89, 595-645 (1988).

2. Blomquist, G. J. \& Bagnères, A. G. Insect hydrocarbons: biology, biochemistry, and chemical ecology. (Cambridge University Press, 2010).

3. Nelson, D. R. and Blomquist, G. J. - Insect waxes. in Waxes: Chemistry, Molecular Biology and Functions (ed. Hamilton, R. J.). 1-90 (Oily Press, 1995).

4. Makki, R., Cinnamon, E. \& Gould, A. P. The development and functions of oenocytes. Annu. Rev. Entomol. 59, 405-425 (2014).

5. Juarez, P., Chase, J. \& Blomquist, G. J. A microsomal fatty acid synthetase from the integument of Blattella germanica synthesizes methyl-branched fatty acids, precursors to hydrocarbon and contact sex pheromone. Arch. Biochem. Biophys. 293, 333-341 (1992).

6. Reed, J. R., Quilici, D. R., Blomquist, G. J. \& Reitz, R. C. Proposed mechanism for the cytochrome P 450-catalyzed conversion of aldehydes to hydrocarbons in the house fly, Musca domestica. Biochemistry 34, 16221-16227 (1995).

7. Gu, P., Welch, W. H., Guo, L., Schegg, K. M. \& Blomquist, G. J. Characterization of a novel microsomal fatty acid synthetase (FAS) compared to a cytosolic FAS in the housefly, Musca domestica. Comp. Biochem. Physiol. B: Biochem. Mol. Biol. 118, 447-456 (1997).

8. Qiu, Y., Tittiger, C., Wicker-Thomas, C., Le Goff, G., Young, S., Wajnberg, E., et al. An insect-specific P450 oxidative decarbonylase for cuticular hydrocarbon biosynthesis. Proc. Natl. Acad. Sci. USA 109, 14858-14863 (2012).

9. Chung, H. \& Carroll, S. B. Wax, sex and the origin of species: dual roles of insect cuticular hydrocarbons in adaptation and mating. BioEssays 37, 822-830 (2015).

10. Holze, H., Schrader, L. \& Büllesbach, J. Advances in deciphering the genetic basis of insect cuticular hydrocarbon biosynthesis and variation. Heredity 126, 219234 (2021).

11. Helmkampf, M., Cash, E. \& Gadau, J. Evolution of the insect desaturase gene family with an emphasis on social Hymenoptera. Mol. Biol. Ecol. 32, 456-471 (2015). 
12. Thomas, M. L. \& Simmons, L. W. Cuticular hydrocarbons are heritable in the cricket Teleogryllus oceanicus. J. Evol. Biol. 21, 801-806 (2008).

13. Wurdack, M., Herbertz, S., Dowling, D., Kroiss, J., Strohm, E., Baur, H., et al. Striking cuticular hydrocarbon dimorphism in the mason wasp Odynerus spinipes and its possible evolutionary cause (Hymenoptera: Chrysididae, Vespidae). Proc. R. Soc. B: Biol. Sci 282, 20151777 (2015).

14. Moris, V. C., Christmann, K., Wirtgen, A., Belokobylskij, S. A., Berg, A., Liebig, W. H., Soon, V., Baur, H., Schmitt, T., Niehuis, O. Cuticular hydrocarbons on old museum specimens of the spiny mason wasp, Odynerus spinipes (Hymenoptera: Vespidae: Eumeninae), shed light on the distribution and on regional frequencies of distinct chemotypes. Chemoecology 31, 311-322 (2021).

15. Chertemps, T., Duportets, L., Labeur, C., Ueda, R., Takahashi, K., Saigo, K. \& Wicker-Thomas, C. A female-biased expressed elongase involved in long-chain hydrocarbon biosynthesis and courtship behavior in Drosophila melanogaster. Proc. Natl. Acad. Sci. USA 104, 4273-4278 (2007).

16. Dembeck, L. M., Böröczky, K., Huang, W., Schal, C., Anholt, R. R. \& Mackay, T. F. Genetic architecture of natural variation in cuticular hydrocarbon composition in Drosophila melanogaster. eLife 4, e09861 (2015).

17. Szafer-Glusman, E., Giansanti, M. G., Nishihama, R., Bolival, B., Pringle, J., Gatti, M. \& Fuller, M. T. A role for very-long-chain fatty acids in furrow ingression during cytokinesis in Drosophila spermatocytes. Curr. Biol. 18, 1426-1431 (2008).

18. Jakobsson, A., Westerberg, R. \& Jacobsson, A. Fatty acid elongases in mammals: their regulation and roles in metabolism. Prog. Lipid Res. 45, 237-249 (2006).

19. Pei, X. J., Fan, Y. L., Bai, Y., Bai, T. T., Schal, C., Zhang, Z. F., Chen, N., Li, S., Liu, T. X. Modulation of fatty acid elongation in cockroaches sustains sexually dimorphic hydrocarbons and female attractiveness. PLoS Biol. 19, e3001330 (2021).

20. Dallerac, R., Labeur, C., Jallon, J. M., Knipple, D. C., Roelofs, W. L. \& WickerThomas, C. A delta 9 desaturase gene with a different substrate specificity is responsible for the cuticular diene hydrocarbon polymorphism in Drosophila melanogaster. Proc. Natl. Acad. Sci. USA 97, 9449-9454 (2000). 
21. Takahashi, A., Tsaur, S. C., Coyne, J. A. \& Wu, C. I. The nucleotide changes governing cuticular hydrocarbon variation and their evolution in Drosophila melanogaster. Proc. Natl. Acad. Sci. USA 98, 3920-3925 (2001).

22. Chertemps, T., Duportets, L., Labeur, C., Ueyama, M. \& Wicker-Thomas, C. A female-specific desaturase gene responsible for diene hydrocarbon biosynthesis and courtship behaviour in Drosophila melanogaster. Insect Mol. Biol. 15, 465-473 (2006).

23. Shirangi, T. R., Dufour, H. D., Williams, T. M. \& Carroll, S. B. Rapid evolution of sex pheromone-producing enzyme expression in Drosophila. PLoS Biol. 7, e1000168 (2009).

24. McKinney, M. K. \& Cravatt, B. F. Structure and function of fatty acid amide hydrolase. Annu. Rev. Biochem. 74, 411-432 (2005).

25. Finet, C., Slavik, K., Pu, J., Carroll, S. B. \& Chung, H. Birth-and-death evolution of the fatty acyl-CoA reductase (FAR) gene family and diversification of cuticular hydrocarbon synthesis in Drosophila. Genome Biol. Evol. 11, 1541-1551 (2019).

26. Pei, X. J., Chen, N., Bai, Y., Qiao, J. W., Li, S., Fan, Y. L. \& Liu, T. X. BgFas1: A fatty acid synthase gene required for both hydrocarbon and cuticular fatty acid biosynthesis in the German cockroach, Blattella germanica (L.). Insect Biochem. Mol. Biol. 112, 103203 (2019).

27. Grigoraki, L., Grau-Bové, X., Yates, H. C., Lycett, G. J. \& Ranson, H. Isolation and transcriptomic analysis of Anopheles gambiae oenocytes enables the delineation of hydrocarbon biosynthesis. eLife 9, e58019 (2020).

28. Wicker-Thomas, C., Garrido, D., Bontonou, G., Napal, L., Mazuras, N., Denis, B., et al. Flexible origin of hydrocarbon/pheromone precursors in Drosophila melanogaster. J. Lipid Res. 56, 2094-2101 (2015).

29. Moriconi, D. E., Dulbecco, A. B., Juárez, M. P. \& Calderón-Fernández, G. M. A fatty acid synthase gene (FASN3) from the integument tissue of Rhodnius prolixus contributes to cuticle water loss regulation. Insect Mol. Biol. 28, 850-861 (2019).

30. Grinsted, L., Bilde, T. \& d'Ettorre, P. Cuticular hydrocarbons as potential kin recognition cues in a subsocial spider. Behav. Ecol. 22, 1187-1194 (2011).

31. de Carvalho Guimarães, I., Cardoso, C. A. L., Caramão, E. B., Schneider, J. K. \& Junior, W. F. A. Analysis of cuticular chemical profiles of Latrodectus geometricus 
(Araneae: Theridiidae) females and juveniles using GC× GC/qMS. Ciência e Natura 40, 1 (2018).

32. Oudejans, R. C. H. M. \& Zandee, D. I. The biosynthesis of the hydrocarbons in males and females of the millipede Graphidostreptus tumuliporus. J. Insect Physiol. 19, 2245-2253 (1973).

33. Zhang, D., Terschak, J. A., Harley, M. A., Lin, J. \& Hardege, J. D. Simultaneously hermaphroditic shrimp use lipophilic cuticular hydrocarbons as contact sex pheromones. PLoS One 6, e17720 (2011).

34. Copeman, L. A., Stoner, A. W., Ottmar, M. L., Daly, B., Parrish, C. C. \& Eckert, G. L. Total lipids, lipid classes, and fatty acids of newly settled red king crab (Paralithodes camtschaticus): comparison of hatchery-cultured and wild crabs. J. Shellfish Res. 31, 153-165 (2012).

35. Romer, F. \& Gnatzy, W. Arachnid oenocytes: ecdysone synthesis in the legs of harvestmen (Opilionidae). Cell Tissue Res. 216, 449-453 (1981).

36. Fontanetti, C. S., Camargo-Mathias, M. I. \& Tiritan, B. The fat body in Rhinocricus padbergi (Diplopoda, Spirobolida). Iheringia. Série Zoologia 94, 351-355 (2004).

37. Symonová, R. \& Smrž, J. First record of hemocytes and oenocytes in freshwater ostracodes. J. Crustac. 29, 18-25 (2009).

38. Feyereisen, R. Origin and evolution of the CYP4G subfamily in insects, cytochrome P450 enzymes involved in cuticular hydrocarbon synthesis. Mol. Phylogenet. Evol. 143, 106695 (2020).

39. Nelson, D. R. Cytochrome P450 diversity in the tree of life. Biochim. Biophys. Acta 1866, 141-154 (2018).

40. Piek, T. Synthesis of wax in the honeybee (Apis mellifera L.). J. Insect Physiol. 10, 563-572 (1964).

41. Katase, H. \& Chino, H. Transport of hydrocarbons by haemolymph lipophorin in Locusta migratoria. Insect Biochem. 14, 1-6 (1984).

42. Billeter, J. C., Atallah, J., Krupp, J. J., Millar, J. G. \& Levine, J. D. Specialized cells tag sexual and species identity in Drosophila melanogaster. Nature 461, 987-991 (2009). 
43. Savarit, F., and Ferveur, J. F. Genetic study of the production of sexually dimorphic cuticular hydrocarbons in relation with the sex-determination gene transformer in Drosophila melanogaster. Genet. Res. 79, 23-40 (2002).

44. Beckmann, H. E. Beeinflussung des Gedächtnisses der Honigbiene durch Narkose, Kühlung und Streß. J. Comp. Physiol. 94, 249-266 (1974).

45. Kovats, V. E. Gas-chromatographische charakterisierung organischer Verbindungen. Teil 1: Retentionsindices aliphatischer Halogenide, Alkohole, Aldehyde und Ketone. Helv. Chim. Acta 41, 1915-1932 (1958).

46. Bolger, A. M., Lohse, M. \& Usadel, B. Trimmomatic: a flexible trimmer for Illumina sequence data. Bioinformatics 30, 2114-2120 (2014).

47. Kajitani, R., Toshimoto, K., Noguchi, H., Toyoda, A., Ogura, Y., Okuno, M., Yabana, M., Harada, M., Nagayasu, E., Maruyama, H., Kohara, Y., Fujiyama, A., Hayashi, T., Itoh, T. Efficient de novo assembly of highly heterozygous genomes from whole-genome shotgun short reads. Genome Res. 24, 1384-1395 (2014).

48. Hoff, K. J., Lange, S., Lomsadze, A., Borodovsky, M. \& Stanke, M. BRAKER1: unsupervised RNA-Seq-based genome annotation with GeneMark-ET and AUGUSTUS. Bioinformatics 32, 767-769 (2016).

49. Krupp, J. J., Kent, C., Billeter, J. C., Azanchi, R., So, A. K. C., Schonfeld, J. A., Smith, B. P., Lucas, C. Levine, J. D. Social experience modifies pheromone expression and mating behavior in male Drosophila melanogaster. Curr. Biol. 18, 1373-1383 (2008).

50. Dobin, A., Davis, C. A., Schlesinger, F., Drenkow, J., Zaleski, C., Jha, S., et al. STAR: ultrafast universal RNA-seq aligner. Bioinformatics 29, 15-21 (2013).

51. Liao, Y., Smyth, G. K. \& Shi, W. featureCounts: an efficient general purpose program for assigning sequence reads to genomic features. Bioinformatics 30, 923930 (2014).

52. Love, M. I., Anders, S. \& Huber, W. Differential analysis of count data - the DESeq2 package. Genome Biol. 15, 550 (2014).

53. Robinson, M. D., McCarthy, D. J. \& Smyth, G. K. edgeR: a Bioconductor package for differential expression analysis of digital gene expression data. Bioinformatics 26, 139-140 (2010). 
54. Finn, R. D., Coggill, P., Eberhardt, R. Y., Eddy, S. R., Mistry, J., Mitchell, A. L., et al. The Pfam protein families database: towards a more sustainable future. Nucleic Acids Res. 44, D279-D285 (2016).

55. Eddy, S. R. Accelerated Profile HMM Searches. PLoS Comput Biol. 7, e1002195 (2011).

56. Katoh, K. \& Standley, D. M. MAFFT multiple sequence alignment software version 7: improvements in performance and usability. Mol. Biol. Evol. 30, 772-780 (2013).

57. Nguyen, L. T., Schmidt, H. A., Von Haeseler, A. \& Minh, B. Q. IQ-TREE: a fast and effective stochastic algorithm for estimating maximum-likelihood phylogenies. Mol. Biol. Evol. 32, 268-274 (2015).

58. Camacho C., Coulouris G., Avagyan V., Ma N., Papadopoulos J., Bealer K. \& Madden T.L. "BLAST+: architecture and applications." BMC Bioinformatics 10, 421 (2008).

59. Johnson, M., Zaretskaya, I., Raytselis, Y., Merezhuk, Y., McGinnis, S. \& Madden, T. L. (2008). NCBI BLAST: a better web interface. Nucleic Acids Res. 36, W5-W9 (2008).

60. Thisse, C. \& Thisse, B. High-resolution in situ hybridization to whole-mount zebrafish embryos. Nat. Protoc. 3, 59-69 (2008).

61. Ye, J., Coulouris, G., Zaretskaya, I., Cutcutache, I., Rozen, S. \& Madden, T. L. Primer-BLAST: a tool to design target-specific primers for polymerase chain reaction. BMC Bioinformatics 13, 134 (2012).

62. Dearden, P. K., Duncan, E. J. \& Wilson, M. J. Fixation and storage of honeybee (Apis mellifera) tissues. Cold Spring Harb. Protoc. 2009, pdb-prot5224 (2009).

63. Dearden, P. K., Duncan, E. J. \& Wilson, M. J. Whole-mount in situ hybridization of honeybee (Apis mellifera) tissues. Cold Spring Harb. Protoc. 2009, pdb-prot5225 (2009).

64. Roma, G. C., Bueno, O. C. \& Camargo-Mathias, M. I. Morpho-physiological analysis of the insect fat body: a review. Micron, 41, 395-401 (2010).

65. Weinstock, G. M., Robinson, G. E., Gibbs, R. A., Worley, K. C., Evans, J. D., Maleszka, R., Robertson, H. M., Weaver, D. B., Beye, M., Bork, P., Elsik, C. G., 
Hartfelder, K., Hunt, G. J., Zdobnov, E. M., Amdam, G. V., Bitondi, M. M. G., Collins, A. M., Cristino, A. S., Lattorff, H. M. G. et al. Insights into social insects from the genome of the honeybee Apis mellifera. Nature 443, 931-949 (2006).

66. Lê, S., Josse, J. \& Husson, F. FactoMineR: an R package for multivariate analysis. J. Stat. Softw. 25, 1-18 (2008).

67. Brereton, R. G. \& Lloyd, G. R. Partial least squares discriminant analysis: taking the magic away. J. Chemom. 28, 213-225 (2014).

68. Liland, K. H. \& Indahl, U. G. Powered partial least squares discriminant analysis. J. Chemom. 23, 7-18 (2009).

69. Mainland, R. L., Lyons, T. A., Ruth, M. M. \& Kramer, J. M. Optimal RNA isolation method and primer design to detect gene knockdown by qPCR when validating Drosophila transgenic RNAi lines. BMC Res. Notes 10, 647 (2017).

70. Pfaffl, M. W. A new mathematical model for relative quantification in real-time RT-PCR. Nucleic Acids Res. 29, E45 (2001). 


\section{Acknowledgments}

We are indebted to Karl-Heinz Schmalz and Wolf-Harald Liebig for providing us $O$. spinipes samples and to Jean-Yves Baugnée for providing information where to find O. spinipes in Belgium. We thank Michael Schnell, Harald Noeske, and Rainer Blum for help with building trap nests. We are thankful to the following people for fruitful discussions, help in the lab, and/or with handling bees and wasps: Rabea Blümel, Dr. Nicolas Braseroa, Dr. Ruth Castillo, Dr. Laura Degirmenci, Janina Diehl, Claudia Etzbauer, Dr. Hermes Escalona, Martin Gabel, Dr. Ewald Grosse-Wilde, Wolf Haberer, Sinan Kaya-Zeeb, Sabine Knaup, Sandra Kukowka, Ruth Lieberth, Dr. Jana Maehner, Dr. Baptiste Martinet, Dr. Christine Missbach, Karin Möller, Maya Nakajima, Dr. Fabian Rüdenauer, Florentine Schaub, Felix Schilcher, Doris Waffler, Katharina Wagler. We are thankful to Mareen Geyer for help with analyzing $\mathrm{CHC}$ profile data. We are grateful to Dr. Daniel Elsner, Dr. Jose Manuel Monroy Kuhn, and Dr. Karen Meusemann for their support regarding differential gene expression analyses. We thank Prof. Dr. Judith Korb and PD Dr. Volker Nehring for advice regarding statistical analyses, and we thank Dr. Thomas Pauli for bioinformatic support. We acknowledge support by the Freiburg Galaxy Team: (i.e., Prof. Rolf Backofen, Bérénice Batut, Bjoern Gruening, Torsten Houwaart), funded by the Collaborative Research Centre 992 Medical Epigenetics (DFG grant SFB 992/1 2012) and German Federal Ministry of Education and Research (BMBF grant 031 A538A RBC). We acknowledge the Struktur- und Genehmigungsbehörde Süd and the Struktur- und Genehmigungsdirektion Nord (both Rhineland Palatinate, Germany) for granting permission to collect samples and Gaby Schöning for granting permission to place trap nests at a bee hotel in Büchelberg. Major parts of the present study were funded by the German Research Foundation (DFG; NI1387/2-1, SCHM 2645/6-1).

\section{Author contribution table}

O.N., T.S., and V.C.M. conceived and designed the project. A.D., B.M., J.W., M.P., O.N., and S.M. sequenced, assembled, and annotated the Odynerus spinipes 
genome. J.P.O. and V.C.M. performed the comparative transcriptomic analyses. L.P. and V.C.M. inferred the gene trees. D.L. and V.C.M. designed the in situ hybridization experiments. M.T., O.N., R.S., T.S., and V.C.M. contributed to the design of the knockdown experiments. V.C.M. conducted the chemotype persistency, the in situ hybridization, the knockdown experiments, and all chemical and statistical analyses. D.L., O.N., R.S., T.S. provided instruments and reagents. O.N. and V.C.M. wrote the manuscript, with contributions from T.S. All authors provided feedback on the manuscript.

\section{Competing Interests statement}

The authors declare no competing financial interests. 


\section{Tables}

Table 1. Cuticular hydrocarbon biosynthesis candidate genes in the mason wasp Odynerus spinipes. The table informs about 1) between which groups differential expression differences (DGE) were detected, 2) orthologous/homologous genes in the honey bee (Apis mellifera) genome, 3) the genes' predicted functions, and 4) the expression of the candidate genes in bee fat body cells.

\begin{tabular}{|c|c|c|c|c|}
\hline $\begin{array}{l}\text { Wasp gene } \\
\text { ID }\end{array}$ & $\begin{array}{l}\text { DGE } \\
\text { between }\end{array}$ & $\begin{array}{l}\text { Honey bee } \\
\text { gene ID }\end{array}$ & Predicted function & Cell type \\
\hline g3158 & age classes & GB52590 & fatty acid synthase & oenocytes (weak) \\
\hline g6537 & age classes & GB46038 & fatty acid elongase & $\begin{array}{l}\text { oenocytes } \\
\text { trophocytes }\end{array}$ \\
\hline g7609 & age classes & GB54399 & fatty acid elongase & oenocytes (weak) \\
\hline g7610 & age classes & GB54397 & fatty acid elongase & oenocytes \\
\hline g7616 & $\begin{array}{l}\text { age classes / } \\
\text { chemotypes }\end{array}$ & GB51247 & fatty acid elongase & oenocytes \\
\hline g7620 & age classes & GB54401 & fatty acid elongase & trophocytes \\
\hline g7621 & age classes & GB54302 & fatty acid elongase & NA \\
\hline g14708 & age classes & $\mathrm{NA}^{1}$ & fatty acid desaturase & oenocytes \\
\hline g14712 & chemotypes & $\begin{array}{l}\text { GB42218 } \\
\text { GB51236 } \\
\text { GB51238 }\end{array}$ & fatty acid desaturase & $\begin{array}{l}\text { oenocytes } \\
\text { oenocytes } \\
\text { trophocytes }\end{array}$ \\
\hline g2413 & age classes & GB50627 & fatty acyl-CoA reductase & oenocytes \\
\hline g1571 & age classes & GB52087 & fatty acyl-CoA reductase & $\begin{array}{l}\text { oenocytes } \\
\text { trophocytes }\end{array}$ \\
\hline g7842 & age classes & GB49380 & fatty acyl-CoA reductase & oenocytes \\
\hline g283 & chemotypes & GB44756 & $\begin{array}{l}\text { rhythmically expressed gene } 5 \\
\text { protein }\end{array}$ & NA \\
\hline g2290 & chemotypes & GB536952 & fatty acid amine hydrolase & $\begin{array}{l}\text { oenocytes } \\
\text { trophocytes (weak) }\end{array}$ \\
\hline g3059 & chemotypes & GB52820 & $\begin{array}{l}\text { parathyroid hormone-related } \\
\text { peptide receptor-like protein }\end{array}$ & $\begin{array}{lc} & \text { (weak) } \\
\text { trophocytes } & \text { (weak) } \\
\text { hexagonal cells [strong] }\end{array}$ \\
\hline
\end{tabular}

\footnotetext{
1 Since we found no 1:1 ortholog in the honey bee genome, we assessed the phylogenetically most closely related homolog,
} GB40659, for being involved in $\mathrm{CHC}$ biosynthesis.

2 One of three co-orthologs in the honey bee, the other two being GB48850 and GB47832 (not studied by us). 
Table 2. Additional cuticular hydrocarbon biosynthesis candidate genes studied by us in the honey bee (Apis mellifera). Given are 1) the fly and 2) the honey bee gene IDs, 3) the genes' predicted functions, and 4) the expression of the candidate genes in honey bee fat body cells.

\begin{tabular}{|c|c|c|c|c|}
\hline $\begin{array}{l}\text { Fly } \\
\text { gene ID }\end{array}$ & $\begin{array}{l}\text { Honey } \\
\text { gene ID }\end{array}$ & bee & Predicted function & Cell type \\
\hline FASN1/2 & GB53412 & & fatty acid synthase & hexagonal cells \\
\hline CG30008 & GB55040 & & fatty acid elongase & oenocytes + trophocytes \\
\hline CG30008 & GB40681 & & fatty acid elongase & hexagonal cells \\
\hline CG5326 & GB54396 & & fatty acid elongase & oenocytes + trophocytes \\
\hline CG31522 & GB51250 & & fatty acid elongase & oenocytes \\
\hline CG2781 & GB54404 & & fatty acid elongase & oenocytes \\
\hline Desat 1,2 , and $\mathrm{F}$ & GB48195 & & fatty acid desaturase & oenocytes + trophocytes \\
\hline
\end{tabular}




\section{List of figures}

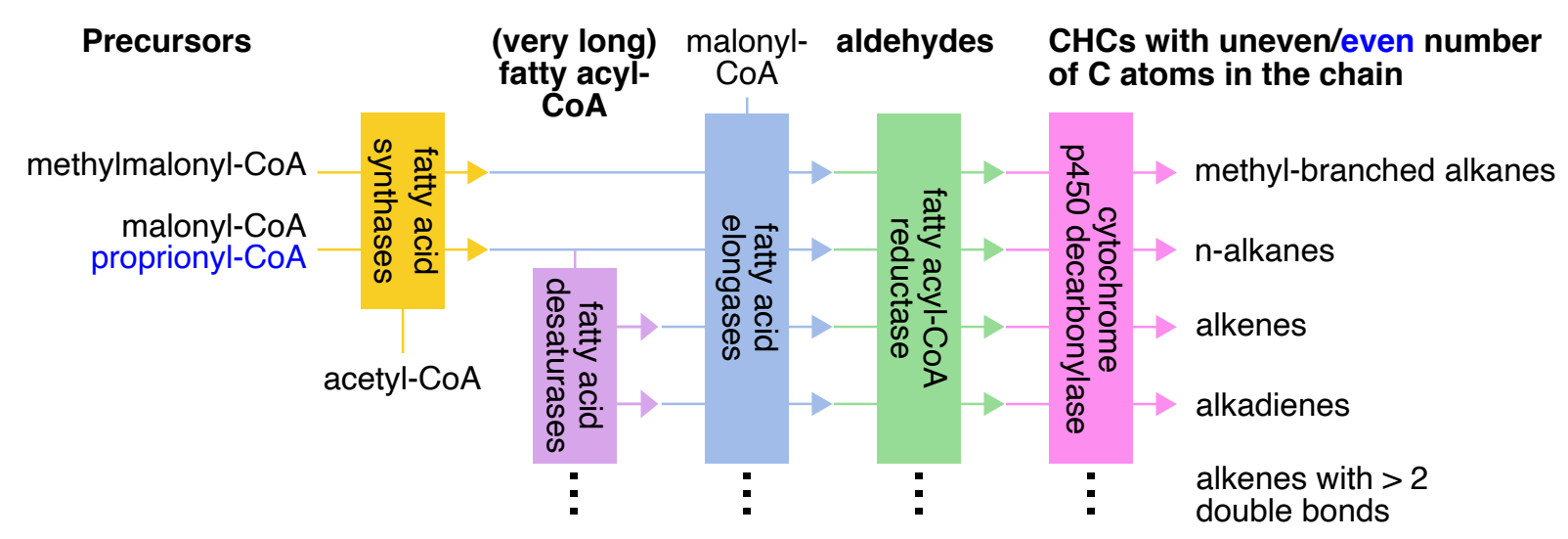

Figure 1. Schematic overview of the main steps of cuticular hydrocarbon biosynthesis. The pathway starts with a fatty acid synthase (FAS) that binds acetyl-coenzyme A (CoA) with malonyl-CoA units. The resulting acyl-CoA (with an even number of carbon atoms) is repeatedly elongated by a FAS which binds acyl-CoA with malonyl-CoA. During each elongation step, the chain length of the fatty acyl-CoA increases by two carbon atoms. Fatty acyl-CoA chains with an uneven number of carbon atoms are thought to arise from substituting malonyl-CoA with propionyl-CoA during the elongation step. The consecutive elongation of the fatty acyl-CoA chain by FAS results in long chain fatty acyl-CoAs. Additional elongation of these fatty acyl-CoA chains to very-long fatty acyl-CoA requires the action of fatty acid elongases that add additional malonyl-CoA molecules to the long chain fatty acyl-CoA. These are reduced into aldehydes by fatty acyl-CoA reductases. Before the latter step, double bonds can be inserted in the (very-)long chain fatty acyl-CoAs by fatty acid desaturases, resulting in the biosynthesis of unsaturated hydrocarbons with a different number of double bonds (alkenes, alkadienes). The biosynthesis of methyl-branched alkanes is thought to depend on the action of a specialized fatty acid synthase that incorporates methylmalonyl-CoA instead of malony-CoA into the chain at specific positions during the chain elongation. The last step of $\mathrm{CHC}$ biosynthesis is the oxidative decarbonylation of aldehydes leading to hydrocarbons (with the loss of one carbon) by a cytochrome p450. Based on 2,5,6,7,8,9. 
Euarthropod clades:

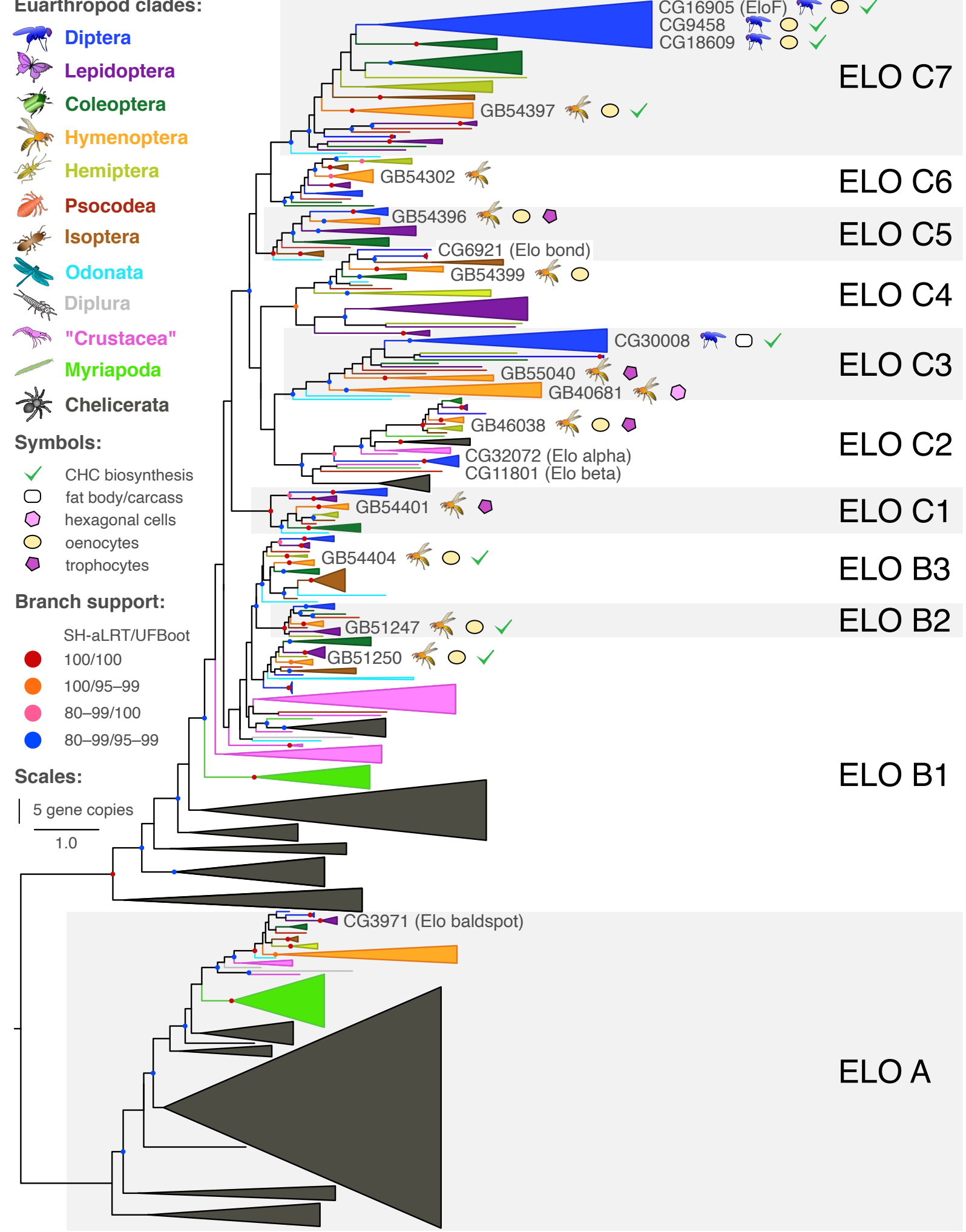

Figure 2. Gene tree of fatty acid elongases from 37 Euarthropoda showing copy numbers and involvement of genes in $\mathrm{CHC}$ biosynthesis. Branch support was calculated from ultrafast bootstrap (UFBoot2) replicates (in \%) and ShimodairaHasegawa approximate likelihood ratio tests ([SH]-aLRT in \%). 


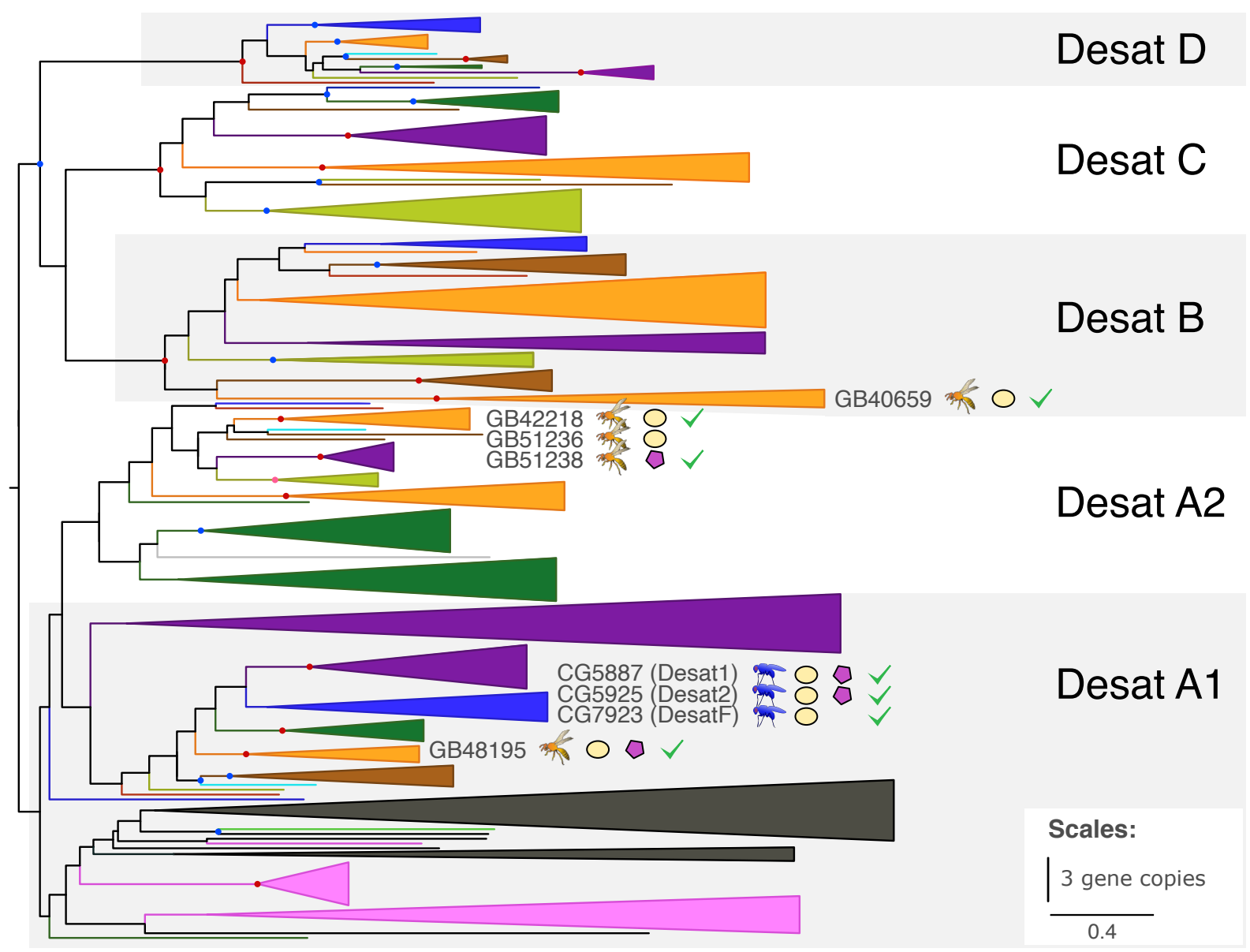

Figure 3. Gene tree of fatty acid desaturases from 37 Euarthropoda. Color coding and symbols are as in the legend of Figure 2. 

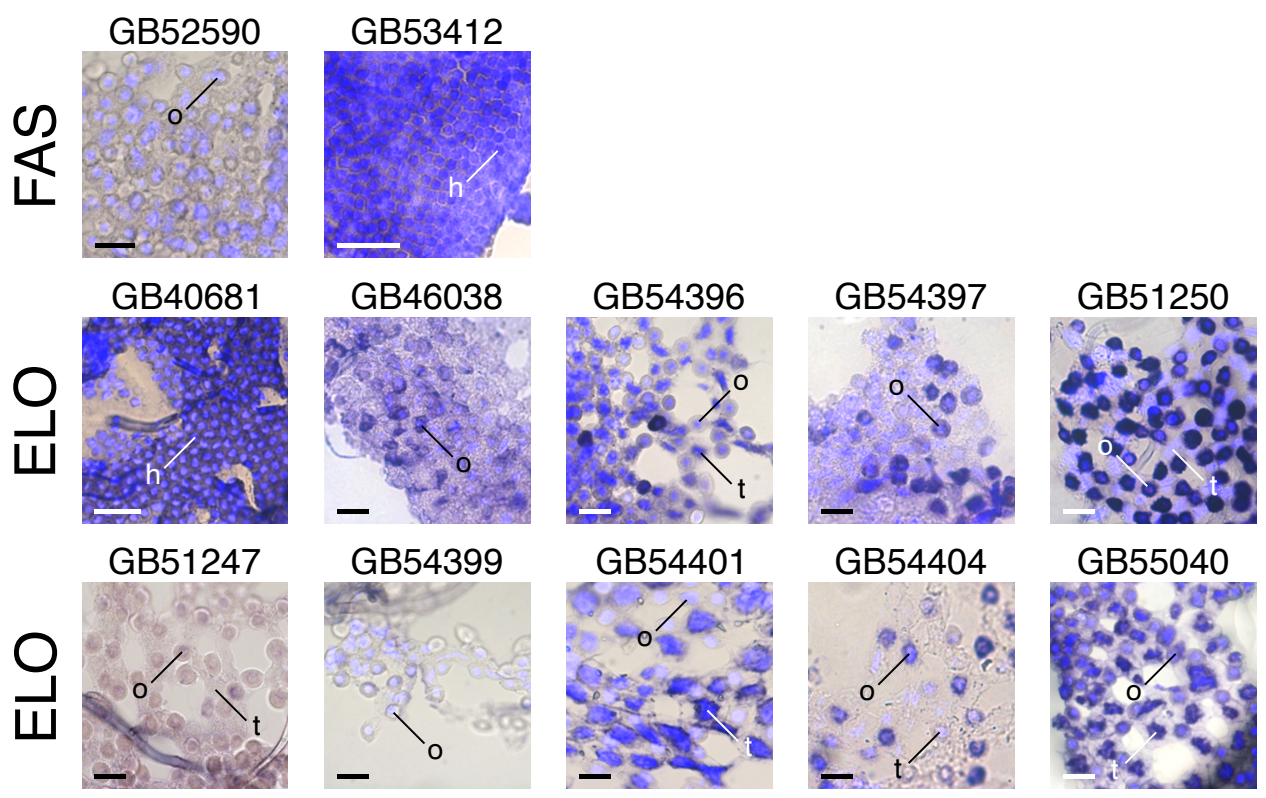

GB54404
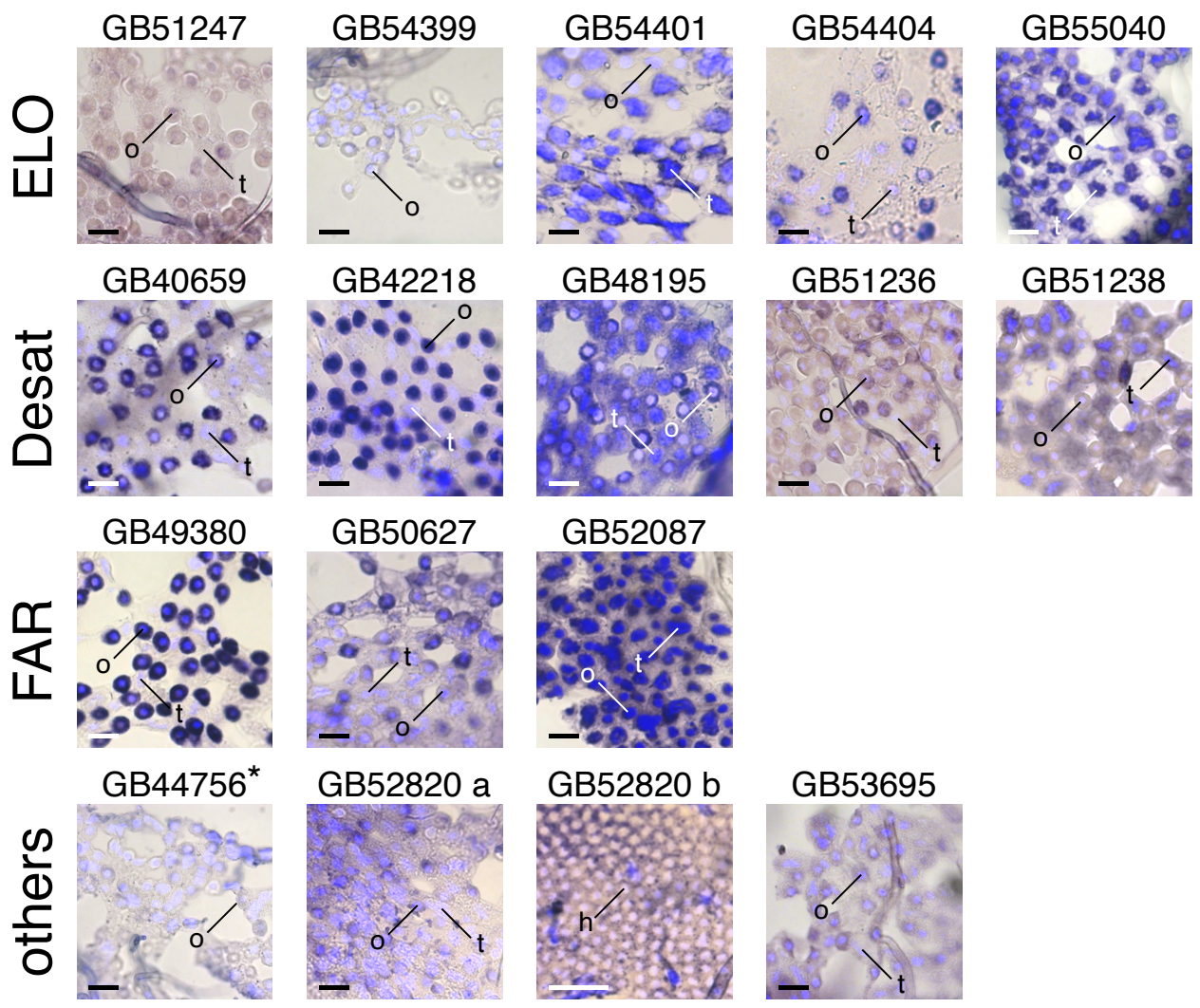

Figure 4. Photomicrographs of worker honey bee (Apis mellifera) fat body cells stained with DAPI (in light blue) and RNA in situ hybridizations (dark blue/purple) visualizing the expression of fatty acid synthases (FAS), fatty acid elongases (ELO), fatty acid desaturases (Desat), fatty acid reductases (FAR), and additional candidate gene transcripts (others). Three fat body cell types are indicated: hexagonal cells $(\mathrm{h})$, oenocytes (o), and trophocytes (t). Two separate photomicrographs visualize the expression of GB52820. DAPI was used to counterstain nuclei. Staining in the photomicrograph marked with an asterisk $\left({ }^{*}\right)$ did not significantly differ from that of the corresponding control (Supplementary Figure 3). Scale bar: $50 \mu \mathrm{m}$. 


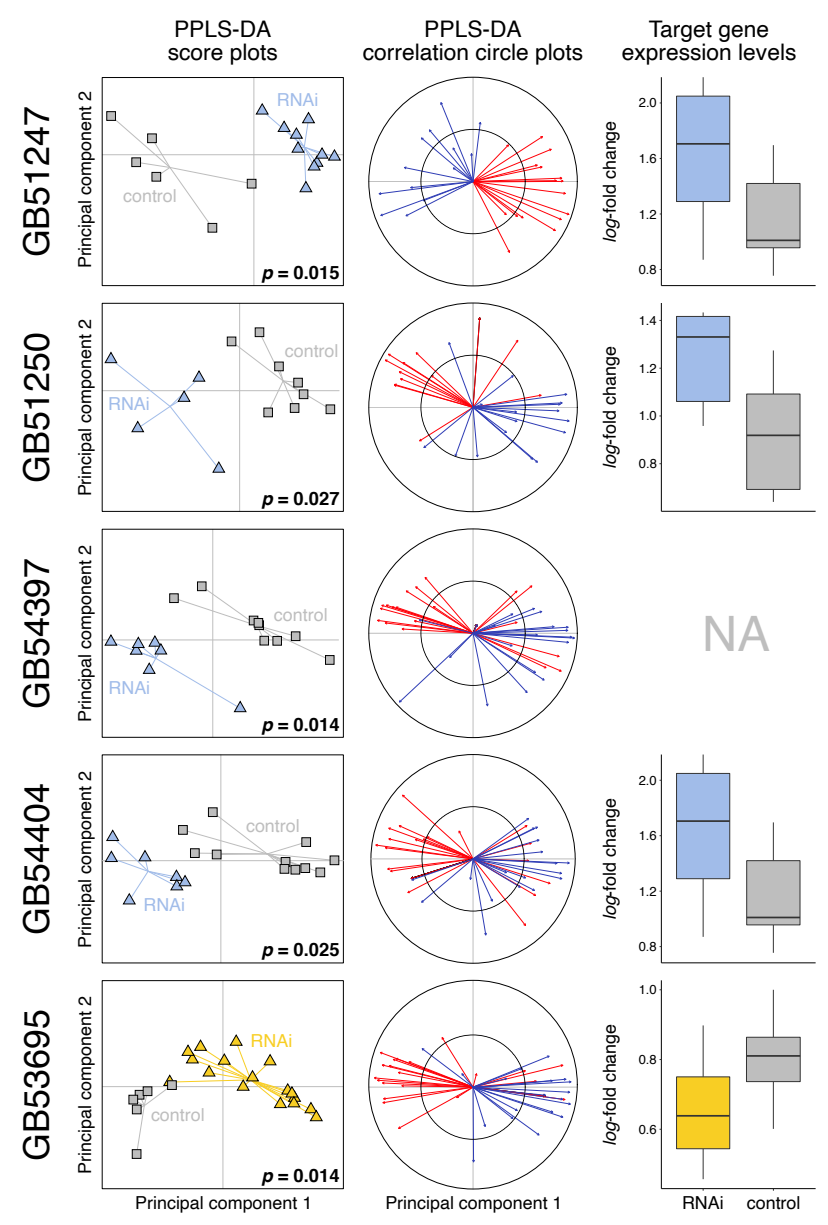

Figure 5. Effects of RNAi-mediated knockdown of four fatty acid elongases (GB51247, GB51250, GB54397, GB54404) and of one fatty acid amide hydrolase (GB53695) in worker honey bees (Apis mellifera) 2-5 days after dsRNA treatment. The first two columns show score plots and correlation circle plots from a powered partial least squares discriminant analysis (PPLS-DA) of the cuticular hydrocarbon $(\mathrm{CHC})$ profile data of target gene-treated and of control bees. $P$-values indicate the statistical probability of group differences representing random variation after BenjaminiHochberg correction for multiple $(N=10)$ testing. The correction circle plots indicate how many $\mathrm{CHCs}$ of a given chain length class (i.e., $\leq 27$ [red] or $>27$ [blue] carbon atoms) correlate with the first two principal components. Box plots in the third column show gene expression levels (log2-fold change) of the target genes in target genetreated and in control bees. None of the expression levels differed statistically significantly between target gene-treated and control bees after applying BenjaminiHochberg correction for multiple testing ( $N=9$; Welch's t-test and Wilcoxon signedrank test). Additional information is shown in Supplementary Figure 4. 


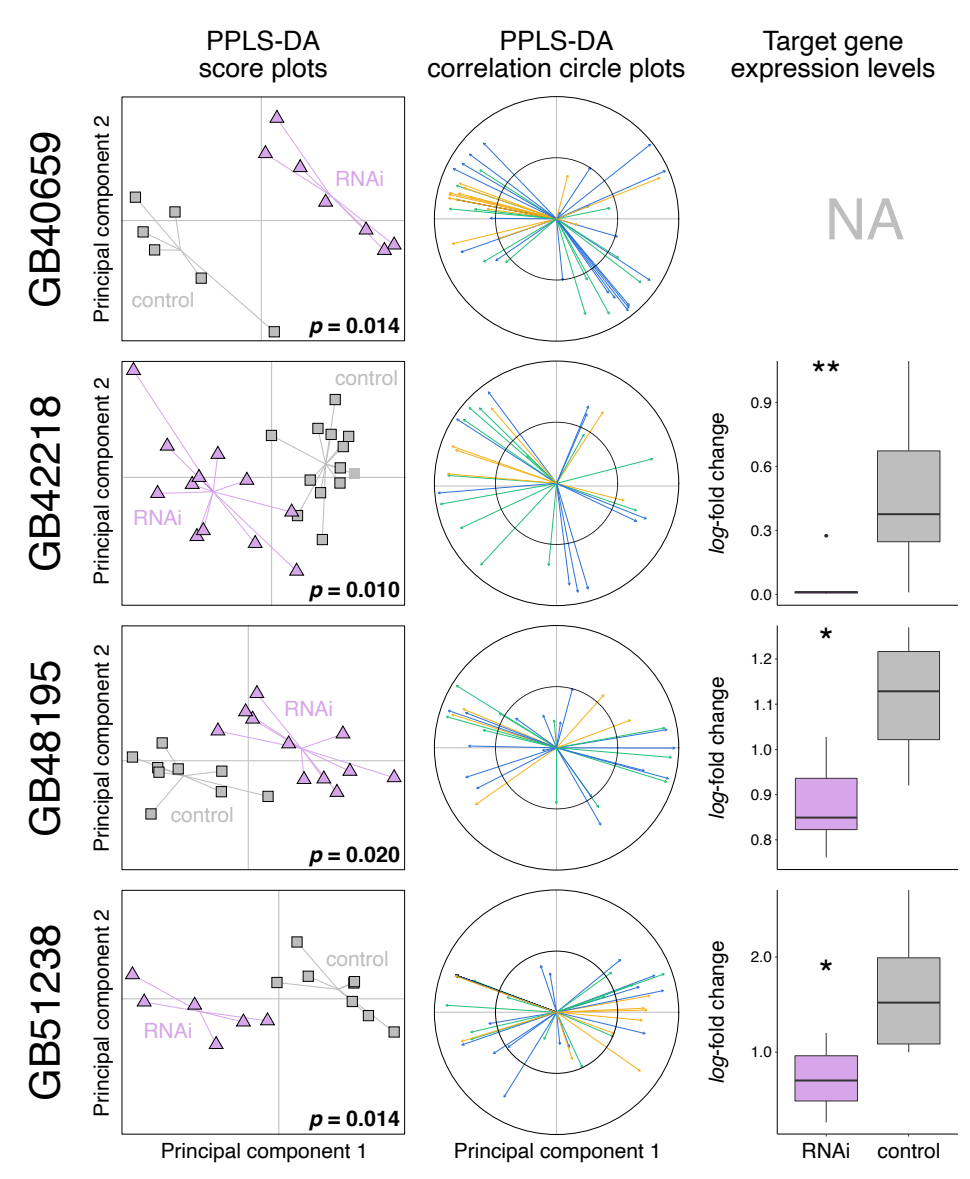

Figure 6. Effects of RNAi-mediated knockdown of four fatty acid desaturases (GB40659, GB48195, GB42218, GB51238) in worker honey bees (Apis mellifera) 2-5 days after dsRNA treatment. The first two columns show score plots and correlation circle plots from a powered partial least squares discriminant analysis (PPLS-DA) of cuticular hydrocarbon $(\mathrm{CHC})$ profile data of target gene-treated and of control bees. $P$ values indicate the statistical probability of group differences representing random variation after Benjamini-Hochberg correction for multiple $(N=10)$ testing. The correction circle plots indicate how many $\mathrm{CHCs}$ of a given compound class (i.e., alkanes [green], alkenes/alkadienes [blue], methyl-branched alkanes [yellow]) correlate with the first two principal components. Box plots in the third column show gene expression levels ( $\log _{2}$-fold change) of the target genes in target gene-treated and in control bees. Asterisks indicate the statistical significance of expression differences between target gene-treated and control bees after applying BenjaminiHochberg correction for multiple testing ( $N=9$; Welch's t-test/Wilcoxon signed-rank test; $\left.p \leq 0.05\left[{ }^{*}\right], p \leq 0.01\left[{ }^{* *}\right]\right)$. Additional information is shown in Supplementary Figure 5. 

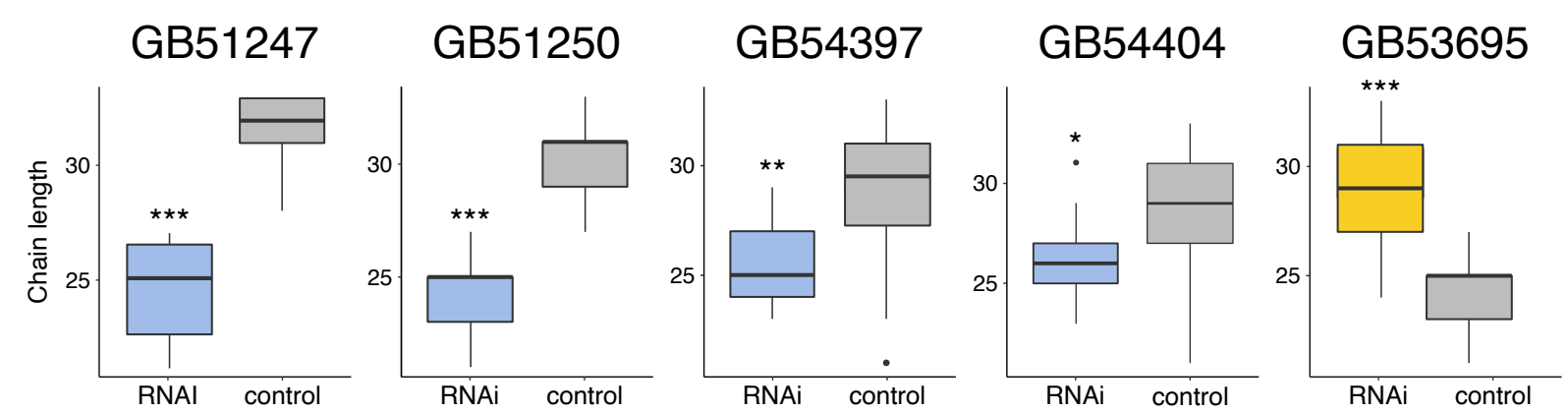

Figure 7. Chain lengths of cuticular hydrocarbons (CHCs) positively associated in a powered partial least squares discriminant analysis along the first principal component with bees treated with dsRNA targeting fatty acid elongases (GB51247, GB51250, GB54397, GB54404; blue) or a fatty acid hydrolase (GB53695; orange) and with bees treated with dsRNA targeting GFP (control; grey). Asterisks indicate statistical significance of abundance differences between the two treatment groups after HolmBonferroni correction for multiple $(\mathrm{N}=5)$ testing (Welch's t-test: $\left.p \leq 0.05{ }^{\star}\right], p \leq 0.01$ $\left.\left[{ }^{* *}\right], p \leq 0.001\left[{ }^{* *}\right]\right)$. 

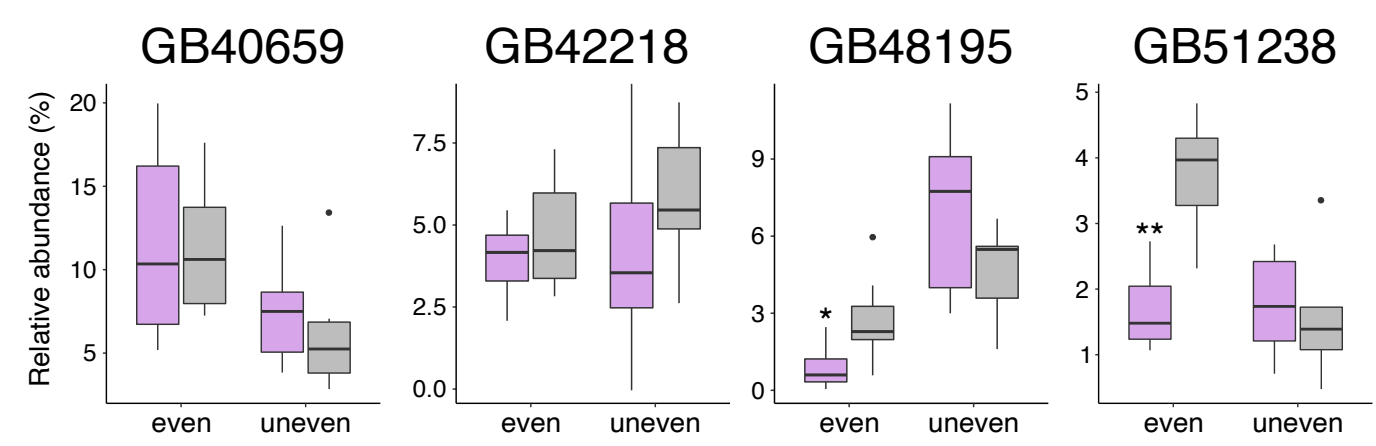

Figure 8. Relative abundance of alkenes with double bonds at even and at uneven positions in bees treated with dsRNA of fatty acid desaturases (pink) or dsRNA of GFP (control; grey). Asterisks indicate statistically significant abundance differences between the two groups after Holm-Bonferroni correction for multiple $(\mathrm{N}=10)$ testing (Welch's t-test/Wilcoxon signed-rank test: $\left.p \leq 0.05\left[{ }^{\star}\right], p \leq 0.01{ }^{* \star}\right], p \leq 0.001\left[{ }^{\star \star \star}\right]$ ). 


\section{Supplementary Files (on Zenodo: 10.5281/zenodo.5552394)}

- Odynerus spinipes draft genome assembly (ODSP_platanus.1.2.4.n0.s20.fa)

- Soft-masked version

of the draft genome (ODSP_platanus.1.2.4.n0.s20.fasta.softmasked)

- Gene models

of predicted

protein-coding

genes (ODSP_platanus.1.2.4.n0.s20_BRAKER1.9_annot.gff)

- mRNA file of predicted gene protein-coding genes (mRNA_ODSP_platanus.1.2.4.n0.s20.fa)

- Protein file of predicted gene prot ein-coding genes (ODSP_BRAKER_augustus_aa.fa)

- Deseq2 results table (age class comparison) (AGE_DESEQ2_sign_results_ordered.xIsx)

- EdgeR results table (age class comparison) (AGE_EdgeR_Irt_sign_results_ordered.xlsx)

- qRT-PCR raw values (Expression_knock_downs_all_Apis_ids.xIsx)

- Cuticular hydrocarbon compound abundances in Apis mellifera samples treated with dsRNA (CHCs_abundance_knock_downs_Apis_ids.xlsx)

- Nucleotide sequence encoding green fluorescent protein (GFP) (Primer_GFP_sequence_used_Negative_contro_RNAi.docx)

- Nucleotide sequence of candidate genes used for probe and dsRNA synthesis (Sequences_ISH_dsRNA_Apis_ids.fa) 